\title{
Dynamical chiral symmetry breaking in gauge theories with extra dimensions
}

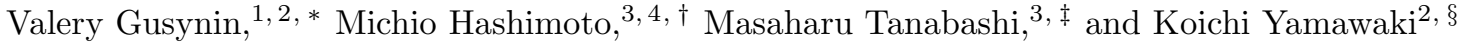 \\ ${ }^{1}$ Bogolyubov Institute for Theoretical Physics, 03143, Kiev, Ukraine \\ ${ }^{2}$ Department of Physics, Nagoya University, Nagoya 464-8602, Japan \\ ${ }^{3}$ Department of Physics, Tohoku University, Sendai 980-8578, Japan \\ ${ }^{4}$ Theory Group, KEK, Oho 1-1, Tsukuba, Ibaraki 305-0801, Japan
}

We investigate dynamical chiral symmetry breaking in vector-like gauge theories in $D$ dimensions with $(D-4)$ compactified extra dimensions, based on the gap equation (Schwinger-Dyson equation) and the effective potential for the bulk gauge theories within the improved ladder approximation. The non-local gauge fixing method is adopted so as to keep the ladder approximation consistent with the Ward-Takahashi identities.

Using the one-loop $\overline{\mathrm{MS}}$ gauge coupling of the truncated KK effective theory which has a nontrivial ultraviolet fixed point (UV-FP) $g_{*}$ for the (dimensionless) bulk gauge coupling $\hat{g}$, we find that there exists a critical number of flavors, $N_{f}^{\text {crit }}(\simeq 4.2,1.8$ for $D=6,8$ for $S U(3)$ gauge theory): For $N_{f}>N_{f}^{\text {crit }}$, the dynamical chiral symmetry breaking takes place not only in the "strong-coupling phase" $\left(\hat{g}>g_{*}\right)$ but also in the "weak-coupling phase" $\left(\hat{g}<g_{*}\right)$ when the cutoff is large enough. For $N_{f}<N_{f}^{\text {crit }}$, on the other hand, only the strong-coupling phase is a broken phase and we can formally define a continuum (infinite cutoff) limit, so that the physics is insensitive to the cutoff in this case.

We also perform a similar analysis using the one-loop "effective gauge coupling". We find the $N_{f}^{\text {crit }}$ turns out to be a value similar to that of the $\overline{\mathrm{MS}}$ case, notwithstanding the enhancement of the coupling compared with that of the $\overline{\mathrm{MS}}$.

PACS numbers: 11.15.Ex,11.10.Kk,11.25.Mj,12.60.Rc

\section{INTRODUCTION}

Much attention has been paid to the extra dimension physics, particularly the large scale scenarios [i, 2. Although the notion of the "extra dimension" might be "deconstructed" in terms of certain

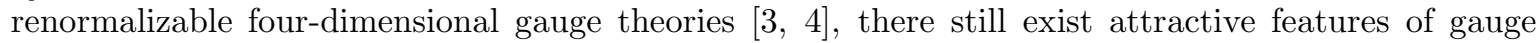
theories with extra dimensions which deserve further non-perturbative studies.

As such three of us [5] (referred to as (I) hereafter) have recently studied dynamical symmetry breaking of the top-mode standard model (TMSM) [6, 7, 8] in a version in $D$ dimensions, with the $D-4$ being compactified extra dimensions (ACDH scenario) [9]. The ACDH scenario was based on earlier proposal 10] of the TMSM with extra dimensions, which was motivated by the topcolor ideas [11], and found that the (dimensionless) bulk QCD coupling above the compactified scale becomes strong due to KK modes contributions and hence may trigger the top quark condensate without ad hoc four-fermion interactions as in the original TMSM. ${ }^{1}$ Unlike Ref. [10] in which only $t_{R}$ is in the bulk, somewhat simpler situation is assumed in the ACDH scenario: the entire third family lives in the bulk, which enables us to investigate dynamically whether the top condensate really takes place in this scenario. In (I) we used the $D$-dimensional gap equation (improved ladder Schwinger-Dyson (SD) equation [13]) with one-loop $\overline{\mathrm{MS}}$ running (bulk) gauge coupling of the truncated KK effective

\footnotetext{
*E-mail: vgusynin@bitp.kiev.ua

${ }^{\dagger}$ E-mail: michioh@tuhep.phvs.tohoku.ac.jp_michioh@post.kek.jp

${ }^{\ddagger}$ E-mail: tanabash@tuhep.phvs.tohoku.ac.jp

$\S$ E-mail: yamawaki@eken.phys.nagoya-u.ac.jp

1 It is discussed in Ref. 12 to combine the original version of TMSM with the space-time having extra dimensions.
} 
theory [14 for such a purpose. ${ }^{2}$ In (I) we found [5] that the (dimensionless) bulk gauge coupling $\hat{g}$ has a nontrivial ultraviolet fixed point (UV-FP) $g_{*}$ in the same one-loop truncated KK effective theory as that ACDH was based on. Since the running bulk gauge coupling rapidly reaches the UV$\mathrm{FP}$, the gap equation is essentially governed by the UV-FP and can well be approximated by that with the running coupling replaced by the UV-FP value: $\hat{g}^{2} \rightarrow g_{*}^{2}$ ("gap equation on the UV-FP"). If we assume the UV-FP persists non-perturbatively, then the bulk QCD coupling is in the weak coupling region $\left(\hat{g}<g_{*}\right)$, since the coupling to be matched with the 3 -brane QCD coupling at the compactification scale is certainly a weak coupling there and hence never exceeds the UV-FP. This implies that top quark condensate is only possible when $g_{*}>\hat{g}_{\text {crit }}\left(\kappa_{D}>\kappa_{D}^{\text {crit }}\right.$ in the notation of (I), with $\kappa_{D}$ proportional to $g_{*}^{2}$ ), where $\hat{g}_{\text {crit }}$ is determined by the SD gap equation just on the UV-FP mentioned above. We then found that top quark condensate cannot take place in the simplest case of the ACDH scenario, $D=6$ and $N_{f}=2$ (only the third family besides gauge bosons lives in the bulk), where we found the UV-FP value is smaller than the critical value $\left(g_{*}<\hat{g}_{\text {crit }}\right)$, while in the case with $D=8$ and $N_{f}=2$ we found it can $\left(g_{*}>\hat{g}_{\text {crit }}\right)$.

In (I) we further studied the phase structure of gauge theories in $D$ dimensions with the $D-4$ dimensions compactified, not restricted to the TMSM. Since the gap equation on the UV-FP possesses a scale invariance, the phase transition takes the form of "conformal phase transition" [16] having an essential-singularity-type scaling. When $g_{*}>\hat{g}_{\text {crit }}$, the dynamical mass function has a slowly damping asymptotic behavior which corresponds to a large anomalous dimension $\gamma_{m}=D / 2-1$, somewhat similar to the walking technicolor [17.

We also discussed in (I) another gap equation, with the one-loop $\overline{\mathrm{MS}}$ running coupling replaced by the one-loop "effective coupling" which includes finite renormalization effects. Since the effective coupling turned out to be considerably enhanced compared with the $\overline{\mathrm{MS}}$ one, we argued that there might exist a possibility that even the simplest case of ACDH scenario with $D=6$ and $N_{f}=2$ may give rise to a top quark condensate.

In this paper, we further study the non-perturbative dynamics of various vector-like gauge theories with extra dimensions, not restricted to the TMSM. Since $g_{*}^{2}$ (or $\kappa_{D}$ ) is written in terms of $N_{f}$, we find that there exists a critical number of flavors $N_{f}^{\text {crit }}$, such that $N_{f}>N_{f}^{\text {crit }}$ for $g_{*}>\hat{g}_{\text {crit }}$. We find that there exists a rich phase structure in such theories: The phase is separated not only into $N_{f}>N_{f}^{\text {crit }}\left(g_{*}>\hat{g}_{\text {crit }}\right)$ and $N_{f}<N_{f}^{\text {crit }}\left(g_{*}<\hat{g}_{\text {crit }}\right)$, but also into $\hat{g}>g_{*}$ (strong coupling phase) and $\hat{g}<g_{*}$ (weak coupling phase) (See Fig.2). This may be useful for a large variety of model buildings beyond the standard model.

In order to systematically study the SD gap equation in a manner consistent with the WardTakahashi identity, we adopt a so-called non-local gauge fixing. Actually, as is known in the fourdimensional case [18], the chiral Ward-Takahashi identity is violated in the gap equation of (I) which is "improved" from the ladder SD equation by a simple ansatz to replace the constant (dimensionful) bulk gauge coupling $g_{D}$ by the running one as 13 :

$$
g_{D}^{2} \rightarrow g_{D}^{2}\left(\max \left(-p^{2},-q^{2}\right)\right)
$$

where $p^{\mu}$ and $q^{\mu}$ are the momenta of external and loop fermions, respectively. This problem can be solved by taking the running coupling as [18]

$$
g_{D}^{2} \rightarrow g_{D}^{2}\left(-(p-q)^{2}\right)
$$

namely a function of gauge boson loop momentum. Then the Landau gauge used in (I) no longer guarantees $A\left(-p^{2}\right) \equiv 1$, which is then inconsistent with the bare vertex ansatz of the ladder approximation. This problem can also be remedied by employing the so-called non-local gauge fixing [18, 19, 20, 21, by which the gauge parameter is arranged to be momentum-dependent so as to keep $A\left(-p^{2}\right) \equiv 1$. Note that the above problems are numerically not serious in the four-dimensional cases and the method of

2 Dynamical chiral symmetry breaking in other types of models with extra dimensions is studied in Ref. [15]. 
(I) is widely used accordingly. However, situation in the higher dimensional case with power running coupling may drastically be changed.

We first re-analyze the gap equation on the UV-FP with $\hat{g} \equiv g_{*}$, in which the dynamical symmetry breaking takes place for $g_{*}>\hat{g}_{\text {crit }}\left(N_{f}>N_{f}^{\text {crit }}\right)$ as in (I): As a result of the above more sophisticated treatment, however, we find that $\hat{g}_{\text {crit }}^{2}$ is larger than that of (I) by a factor $D / 4$, which is a substantial change for $D \gg 4$. This result implies that the dynamical symmetry breaking gets suppressed compared with the result of (I). For the $S U(3)$ gauge theory our new gap equation yields:

$$
N_{f}^{\text {crit }} \simeq \begin{cases}4.2, & \text { for } D=6 \\ 1.8, & \text { for } D=8 \\ 0.8, & \text { for } D=10\end{cases}
$$

Based on the gap equation both on and off the $U V-F P$, we further reveal a full phase structure in the $\hat{g}^{2}-N_{f}$ plane: Although the solution of the gap equation on the $U V-F P$, as in the analysis of (I), just separates the phases by $N_{f}>N_{f}^{\text {crit }}\left(g_{*}>\hat{g}_{\text {crit }}\right)$ and $N_{f}<N_{f}^{\text {crit }}\left(g_{*}<\hat{g}_{\text {crit }}\right)$, we here also analyze the gap equation off the $U V$ - $F P$ which further separates the phases by $\hat{g}>g_{*}$ ("strong-coupling phase") and $\hat{g}<g_{*}$ ("weak-coupling phase").

For $N_{f}>N_{f}^{\text {crit }}\left(g_{*}>\hat{g}_{\text {crit }}\right)$, which is the case we studied in (I), the dynamical chiral symmetry breaking takes place not only in the strong-coupling phase $\left(\hat{g}>g_{*}\right)$ but also in the weak-coupling one $\left(\hat{g}<g_{*}\right)$ as far as the cutoff $\Lambda$ is large enough (namely, $\hat{g}\left(\mu=\Lambda\right.$ ) is rather close to $\left.g_{*}\right)$. This case is relevant to the TMSM with extra dimensions (ACDH scenario) [9] whose bulk QCD coupling is matched with that of the brane QCD at the compactification scale which is certainly weak and hence the theory necessarily should be set in the weak-coupling phase. In order to have dynamical symmetry breaking even in the weak-coupling phase, we need to arrange $N_{f}>N_{f}^{\text {crit }}$ : From the result Eq.(3) we conclude that the simplest version of the ACDH scenario with $N_{f}=2$ does not give rise to a top quark condensate for $D=6$, while it can for $D=8$ and $D=10$.

For $N_{f}>N_{f}^{\text {crit }}$, we further find it impossible to define the continuum limit, despite the fact that the essential-singularity type scaling with respect to $\kappa_{D}$ found in (I) superficially suggests a conformal phase transition having a large anomalous dimension $\gamma_{m}=D / 2-1$ : Actually the value of $\kappa_{D}$ is not continuous and hence cannot be taken arbitrarily close to $\kappa_{D}^{\text {crit }}$. Then the UV cutoff should be considered as a physical one and the low-energy physics remains cutoff-sensitive in this case.

Moreover, in the ACDH scenario the scale of the physical UV cutoff $\Lambda$ is no longer an adjustable parameter but a "predictable one" in contrast to the treatment in Ref. [9], since the bulk gauge coupling $\hat{g}$ is completely controlled by the 3-brane QCD coupling at the compactification scale and the KK effective theory, and hence $\Lambda$ is uniquely tied up with the dynamical mass of the condensed fermion (top quark) through the gap equation. If we use as an input the value of $F_{\pi}(\simeq 250 \mathrm{GeV})$ which is also tied up with the top quark mass, then the cutoff is "predicted" in terms of $F_{\pi}$. The situation is completely different from the original TMSM where $\Lambda$ is related through the gap equation only to two parameters: the dynamical mass and the four-fermion coupling which is a free parameter. This implies that even when the model is arranged as $N_{f}>N_{f}^{\text {crit }}$, the phenomenological analysis of Ref. [9] should be modified substantially by taking account of this fact, which we shall report in a separate paper.

For $N_{f}<N_{f}^{\text {crit }}\left(g_{*}<\hat{g}_{\text {crit }}\right)$, on the other hand, we find a novel situation: The strong-coupling phase $\left(\hat{g}>g_{*}\right)$ is in the chiral-symmetry broken phase, while the weak-coupling phase $\left(\hat{g}<g_{*}\right)$ is in the unbroken one, and we can formally define a continuum limit (infinite cutoff limit) at the phase boundary $\hat{g}=g_{*}$ with a large anomalous dimension $\gamma_{m}=(D / 2-1)(1-\tilde{\nu})\left(0<\tilde{\nu} \equiv \sqrt{1-\left(g_{*} / \hat{g}_{\text {crit }}\right)^{2}}<\right.$ 1), a situation similar to the ladder QED [22]. This fact implies that the low-energy physics becomes insensitive to the details of the physics around cutoff (stringy physics ?). Then, no matter whatever physics may exist at the cutoff, the strong-coupling phase of this case may be useful within the framework of local field theory (without referring to, e.g., stringy physics) for model building, such as a "bulk technicolor". The bulk technicolor then resembles the walking technicolor [17] with large anomalous dimension and hence is expected to be free from FCNC problems. 
We also perform a similar analysis using the one-loop "effective gauge coupling". Although the gauge boson propagator explicitly depends on the UV cutoff and cannot be renormalized in this scheme, we find a critical $N_{f}$ turns out to be a value similar to that of the $\overline{\mathrm{MS}}$ case, $4<N_{f}^{\text {crit }}<5$ $(D=6)$. This is rather surprising, considering the fact that as we showed in (I) the effective coupling is enhanced roughly double compared with that of the $\overline{\mathrm{MS}}$. Then this result strongly suggests that for all ambiguities of the approximations of the gap equation, the simplest case of ACDH scenario with $N_{f}=2$ is quite unlikely for $D=6$.

The paper is organized as follows: In Section 2 we write down the SD gap equation in $D$ dimensions with the non-local gauge fixing. In Section 3 we obtain analytical solution to the SD equation on the UV-FP with the running coupling set to be just on the UV-FP, $\hat{g} \equiv g_{*}$. The ground state is identified through the Cornwall-Jackiw-Tomboulis (CJT) effective potential [23]. In Section 4 we present a full phase structure in $\hat{g}^{2}-N_{f}$ plane, based on the solution of the gap equation both on and off the UV-FP: For $N_{f}>N_{f}^{\text {crit }}$ we find that both the strong-coupling phase $\left(\hat{g}>g_{*}\right)$ and the weak coupling phase $\left(\hat{g}<g_{*}\right)$ are broken phases and is relevant to the ACDH scenario of the TMSM whose model building is then constrained by the value of $N_{f}^{\text {crit }}$. We also find no continuum limit in this case and the cutoff is predictable in terms of $F_{\pi} \simeq 250 \mathrm{GeV}$. On the other hand, for $N_{f}<N_{f}^{\text {crit }}$ we find that the UV-FP separates a broken phase (for strong-coupling phase) and an unbroken phase (for the weak coupling phase): We can formally define a continuum limit at the UV-FP with large anomalous dimension and the theory may be useful for "bulk technicolor". In Section 5 we analyze the gap equation with the effective coupling instead of the $\overline{\mathrm{MS}}$ running coupling through the non-local gauge fixing. We also find a mean-field scaling. Section 6 is for Summary and Discussions. Appendix A is devoted to yet another effective potential than the CJT potential, which has a more direct relevance to the bound states picture. In Appendix B we present a Schrödinger-like equation which yields some intuition on the $D$-dependence of the phase transition. Effects of the infrared cutoff in the gap equation are discussed in Appendix Q. Appendix D gathers formulas of effective coupling.

\section{GAP EQUATION WITH THE NON-LOCAL GAUGE FIXING}

Although the $D$-dimensional Lorentz symmetry is explicitly violated by the compactification of the extra dimensions, such a effect should be proportional to the inverse of the compactification radius $R^{-1}$. For sufficiently large momentum $\left|p^{2}\right| \gg R^{-2}$, we thus expect that the $D$-dimensional Lorentz symmetry is restored approximately, which enables us to make an ansatz for the bulk fermion propagator in a Lorentz covariant form:

$$
i S^{-1}(p)=A\left(-p^{2}\right) p-B\left(-p^{2}\right) .
$$

The appearance of the non-zero fermion mass (gap) $B\left(-p^{2}\right) \neq 0$ is a signal of the chiral symmetry breaking in the bulk. The aim of this section is to construct an appropriate gap equation, by which we investigate the chiral phase transition in the vector-like gauge theories with the extra dimensions.

Let us start with the naive ladder approximation of the Schwinger-Dyson equation of the bulk fermion propagator [24]

$$
\begin{aligned}
A\left(-p^{2}\right)=1+ & \frac{C_{F}}{-p^{2}} \int \frac{d^{D} q}{(2 \pi)^{D} i} \frac{A\left(-q^{2}\right)}{-A^{2}\left(-q^{2}\right) q^{2}+B^{2}\left(-q^{2}\right)} \\
& \times\left[-(3-D-\xi) \frac{p \cdot q}{(p-q)^{2}}+2(1-\xi) \frac{p \cdot(p-q) q \cdot(p-q)}{(p-q)^{4}}\right] g_{D}^{2}, \\
B\left(-p^{2}\right)=m_{0}+ & C_{F} \int \frac{d^{D} q}{(2 \pi)^{D} i} \frac{B\left(-q^{2}\right)}{-A^{2}\left(-q^{2}\right) q^{2}+B^{2}\left(-q^{2}\right)} \cdot \frac{(D-1+\xi) g_{D}^{2}}{-(p-q)^{2}}
\end{aligned}
$$

with $C_{F}$ being the Casimir of the fermion representation $\left(C_{F}=\left(N^{2}-1\right) /(2 N)\right.$ for the fundamental representation of $S U(N)$ gauge group). Here $g_{D}, \xi$ and $m_{0}$ are the bulk gauge coupling strength, the 
gauge fixing parameter and the fermion bare mass, respectively. It should be noted that the mass dimension of the gauge coupling strength $g_{D}^{2}$ is negative, $-\delta$, for $D=4+\delta>4$.

Within the naive ladder approximation, the effect of the running gauge coupling strength is completely ignored, however. In order to remedy this drawback, the gauge coupling constant $g_{D}$ needs to be replaced by something in which the running effect is incorporated appropriately.

Since there exist 3 different momenta, $x \equiv-p^{2}, y \equiv-q^{2}, z \equiv-(p-q)^{2}$, in the Schwinger-Dyson Eqs.(5) and (6), there exist various ways to incorporate the running effect in the gap equation. In Ref. [5], we improved the Schwinger-Dyson equation using a simple replacement [13],

$$
g_{D}^{2} \rightarrow g_{D}^{2}(\max (x, y))
$$

with $g_{D}^{2}(\max (x, y))$ being the running gauge coupling. This prescription is widely used and has various technical advantages: The angular integrals in the gap equation can be performed in an analytical manner. The wave function factor $A$ is shown to be unity in the Landau gauge $\xi=0$, which makes the ladder approximation consistent with the vector Ward-Takahashi identity.

Although the prescription Eq.(7) has been used widely in the analysis of the dynamical chiral symmetry breaking in the four dimensional QCD, it is pointed out [18] that Eq. (77) is not consistent with the chiral Ward-Takahashi identity, if the same prescription is applied to the axial-vector vertex.

In this paper, we therefore use a different choice [18]

$$
g_{D}^{2} \rightarrow g_{D}^{2}(z),
$$

in which the gauge boson momentum $z$ is used as the scale of the running gauge coupling strength. The prescription Eq.(8) is consistent with the chiral Ward-Takahashi identity, but it induces nontrivial wave function factor $A$ within conventional gauge fixing methods, leading to an inconsistency with the ladder approximation and the vector Ward-Takahashi identity.

In order to avoid such a dilemma, we use the non-local gauge fixing method. The method was originally invented in the analysis of four dimensional gauge theories [19] and extended to gauge theories in $D$ dimensions [20]. It was then reformulated into a compact formula in the analysis of four dimensional QCD by using a different approach [18]. The method of Ref. [18] is extended to gauge theories in arbitrary dimensions [21]. Here we give a brief derivation of the non-local gauge in order to explain notations used in this paper.

The non-local gauge fixing method is based on the observation that the parameter $\xi$ can be generalized to a function of the momentum $z, \xi(z)$ by introducing the non-local gauge fixing operator. It is then possible to choose the specific form of $\xi(z)$ so as to make the wave function factor $A \equiv 1$.

We start with the Schwinger-Dyson equation of the fermion wave function A, Eq.(5). After the Wick rotation, it reads

$$
A(x)=1+\frac{C_{F}}{x} \int_{0}^{\Lambda^{2}} d y y^{D / 2-1} \frac{A(y)}{A^{2}(y) y+B^{2}(y)} K_{A}(x, y) .
$$

We introduced the ultraviolet cutoff $\Lambda$, where the $D$-dimensional effective field theory is considered to be replaced by yet unknown underlying physics (e.g., string theory, (de)constructed extra dimensions [3, 4], etc.).

The integral kernel $K_{A}$ is given by

$$
\begin{aligned}
K_{A}(x, y)=\frac{\Omega_{\mathrm{NDA}}}{B\left(\frac{1}{2}, \frac{D}{2}-\frac{1}{2}\right)} & \int_{0}^{\pi} d \theta(\sin \theta)^{D-2} g_{D}^{2}(z) \\
& \times\left[(D-1-\xi(z)) \frac{\sqrt{x y} \cos \theta}{z}-2(1-\xi(z)) \frac{x y}{z^{2}} \sin ^{2} \theta\right],
\end{aligned}
$$

with $\Omega_{\mathrm{NDA}}$ being the naive dimensional analysis [25] (NDA) factor

$$
\Omega_{\mathrm{NDA}} \equiv \frac{1}{(4 \pi)^{D / 2} \Gamma(D / 2)}
$$


The angle $\theta$ is the angle between Euclidean momenta $q_{E}$ and $p_{E}$,

$$
z=x+y-2 \sqrt{x y} \cos \theta .
$$

Noting

$$
(\sin \theta)^{D-2} \cos \theta=\frac{1}{D-1} \frac{d}{d \theta}\left((\sin \theta)^{D-1}\right),
$$

we find

$$
\begin{aligned}
K_{A}(x, y)= & \frac{\Omega_{\mathrm{NDA}}}{B\left(\frac{1}{2}, \frac{D}{2}-\frac{1}{2}\right)}\left\{-\frac{1}{D-1} \int_{0}^{\pi} d \theta(\sin \theta)^{D-1} \sqrt{x y} \frac{d}{d \theta}\left((D-1-\xi(z)) g_{D}^{2}(z) \frac{1}{z}\right)\right. \\
& \left.-2 \int_{0}^{\pi} d \theta(\sin \theta)^{D}(1-\xi(z)) g_{D}^{2}(z) \frac{x y}{z^{2}}\right\}
\end{aligned}
$$

where we have integrated $d / d \theta$ by parts.

The $\theta$ differentiation $d / d \theta$ in Eq.(13) can be written as

$$
\frac{d}{d \theta}=\frac{d z}{d \theta} \frac{d}{d z}=2 \sqrt{x y} \sin \theta \frac{d}{d z} .
$$

We then obtain

$$
\begin{aligned}
& K_{A}(x, y)=-2 \frac{\Omega_{\mathrm{NDA}}}{B\left(\frac{1}{2}, \frac{D}{2}-\frac{1}{2}\right)} x y \int_{0}^{\pi} d \theta(\sin \theta)^{D} \\
& \times\left[\frac{1}{D-1} \frac{d}{d z}\left((D-1-\xi(z)) \frac{g_{D}^{2}(z)}{z}\right)+(1-\xi(z)) \frac{g_{D}^{2}(z)}{z^{2}}\right] .
\end{aligned}
$$

The condition $K_{A} \equiv 0$ can be guaranteed if $\xi(z)$ satisfies a differential equation

$$
\frac{1}{D-1} \frac{d}{d z}\left((D-1-\xi(z)) \frac{g_{D}^{2}(z)}{z}\right)+(1-\xi(z)) \frac{g_{D}^{2}(z)}{z^{2}}=0 .
$$

It is easy to solve Eq. (15). We find the solution (non-local gauge) is given by

$$
\xi(z)=\frac{D-1}{g_{D}^{2}(z) z^{D-2}} \int_{0}^{z} d z z^{D-2} \frac{d}{d z} g_{D}^{2}(z),
$$

where the integration constant is taken so as to make $\xi(z)$ regular at $z=0$.

Using the non-local gauge fixing parameter Eq.(16), the wave function factor $A$ can be set unity. The gap equation (6) then reads,

$$
B(x)=m_{0}+C_{F} \int_{0}^{\Lambda^{2}} d y y^{D / 2-1} \frac{B(y)}{y+B^{2}(y)} K_{B}(x, y),
$$

where

$$
K_{B}(x, y) \equiv \frac{\Omega_{\mathrm{NDA}}}{B\left(\frac{1}{2}, \frac{D}{2}-\frac{1}{2}\right)} \int_{0}^{\pi} d \theta(\sin \theta)^{D-2} \frac{(D-1+\xi(z)) g_{D}^{2}(z)}{z} .
$$

Eqs.(16), (17) and (18) are our basic equations to be solved in this paper.

It should be kept in mind that the gap equation Eq. (17) is not valid for $x, y \lesssim R^{-2}\left(x, y \equiv\left|p^{2}\right|,\left|q^{2}\right|\right)$ due to the compactification of the extra dimensions. In order to estimate uncertainties coming from the compactification sensitive infrared region, we introduce an infrared cutoff $M_{0}^{2} \sim R^{-2}$ on and off in the following analyses. We will actually find that many results are insensitive to $M_{0}^{2}$ if the ultraviolet cutoff is taken to be large sufficiently $\Lambda^{2} \gg M_{0}^{2}$. 


\section{SOLUTION AT THE FIXED POINT}

We next consider the running of the gauge coupling in theories with extra dimensions compactified to an orbifold $T^{\delta} / Z_{n}$ with radius $R$. Here $Z_{n}$ represents the discrete group with order of $n$.

In Ref. [5], the dimensionless bulk gauge coupling $\hat{g}$ is defined as,

$$
\hat{g}^{2} \equiv \frac{(2 \pi R \mu)^{\delta}}{n} g^{2},
$$

with $g$ being the gauge coupling of the truncated KK effective theory [14]. The bulk gauge coupling $g_{D}$ is given by

$$
g_{D}^{2}=\frac{\hat{g}^{2}(\mu)}{\mu^{\delta}} .
$$

It is then shown that the dimensionless bulk gauge coupling obeys the renormalization group equation (RGE),

$$
\mu \frac{d}{d \mu} \hat{g}=\frac{\delta}{2} \hat{g}+(1+\delta / 2) \Omega_{\mathrm{NDA}} b^{\prime} \hat{g}^{3},
$$

at the one-loop approximation of the $\overline{\mathrm{MS}}$ coupling of the truncated KK effective theory. The RGE factor $b^{\prime}$ is given by

$$
b^{\prime}=-\frac{26-D}{6} C_{G}+\frac{\eta}{3} T_{R} N_{f},
$$

where $\eta$ represents the dimension of the spinor representation of $S O(1, D-1)$,

$$
\eta \equiv \operatorname{tr}_{\Gamma} 1=2^{D / 2} \quad \text { for even } D,
$$

and $N_{f}$ is the number of fermions in the bulk. The group-theoretical factor $C_{G}$ and $T_{R}$ are given by $C_{G}=N$ and $T_{R}=1 / 2$ for $S U(N)$ gauge theory.

It is interesting to note that the dimensionless gauge coupling $\hat{g}$ has a non-trivial asymptotically stable ultraviolet fixed point (UV-FP),

$$
g_{*}^{2} \Omega_{\mathrm{NDA}}=\frac{1}{-\left(\frac{2}{\delta}+1\right) b^{\prime}},
$$

for $b^{\prime}<0$ or

$$
N_{f}<N_{f}^{\mathrm{ANS}} \equiv \frac{(26-D) C_{G}}{2 \eta T_{R}} .
$$

On the other hand, the coupling $\hat{g}$ grows without a bound in the high energy region (asymptoticallynot-stable) and the UV-FP disappears for $b^{\prime}>0$ or $N_{f}>N_{f}^{\text {ANS }}$. Hereafter we thus restrict ourselves to the analysis of gauge theories with $N_{f}<N_{f}^{\text {ANS }}$.

It is straightforward to solve RGE Eq.(21). Particularly, the coupling $\hat{g}^{2}$ behaves as

$$
\hat{g}^{2}\left(\mu^{2}\right)=\frac{\mu^{2} g_{*}^{2}}{\mu^{2}-\left(\Lambda \frac{(D)}{\mathrm{MS}}\right)^{2}},
$$

in $D=4+2$ dimensional gauge theories. Here $\left(\Lambda \frac{(D)}{\mathrm{MS}}\right)^{2}$ is the scale parameter of the theory. Vanishing of $\left(\Lambda \frac{(D)}{\mathrm{MS}}\right)^{2}=0$ corresponds to the UV-FP solution $\left(\hat{g}^{2}=g_{*}^{2}\right)$ and it implies that the theory 


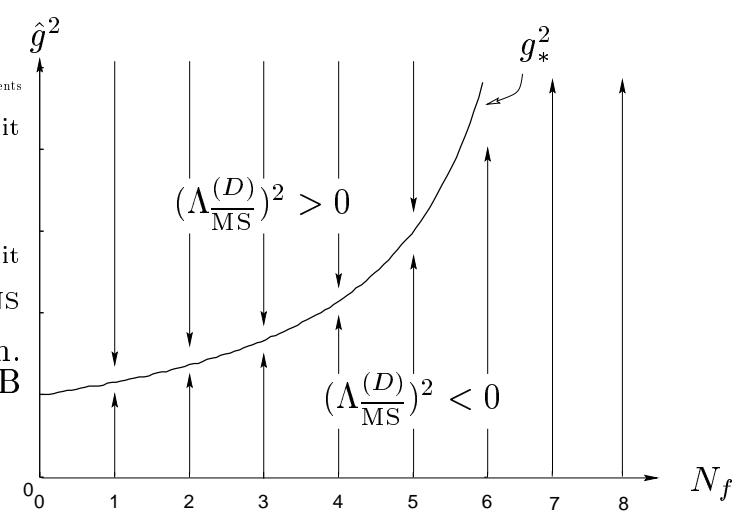

FIG. 1: The one-loop renormalization group flow of $\hat{g}^{2}$ in $S U(3)$ gauge theory in $D=4+2$ dimensions for various $N_{f}$. The gauge coupling $\hat{g}$ approaches its UV-FP for $\mu \rightarrow \infty$.

becomes approximately scale-invariant, except that the scale-invariance is violated by the cutoff $\Lambda$ and the compactification scale $R^{-1}$. On the other hand, positive $\left(\Lambda \frac{(D)}{\mathrm{MS}}\right)^{2}>0$ (negative $\left.\left(\Lambda \frac{(D)}{\mathrm{MS}}\right)^{2}<0\right)$ corresponds to strongly interacting phase $\hat{g}^{2}>g_{*}^{2}$ (weakly interacting phase $\hat{g}^{2}<g_{*}^{2}$ ). See Figure 1 .

Although the renormalization group structure calculated above in the one-loop level cannot be justified within a perturbative analysis, the existence of the non-trivial UV-FP is supported by a recent lattice calculation in a certain case. 26] Moreover, the existence of such a UV-FP may open an interesting possibility in the model building with the compactified extra dimensions. Absence of such a non-trivial UV-FP is implicitly assumed in the conventional models with extra dimensions, in which physical UV cutoff needs to be introduced and the predictions depend on the non-field-theoretical physics at the cutoff scale (e.g., stringy physics). On the other hand, if there exists a non-trivial UV-FP in the model with extra dimensions, the low energy physics can be predicted almost entirely from the field theoretical properties of the UV-FP. The low energy predictions become insensitive to the physics at the UV cutoff.

We believe that this new possibility is interesting enough to justify the investigation of the dynamical properties of the chiral phase transition around the presumed UV-FP. It should be noted, however, the value of the one-loop UV-FP Eq.(24) can be affected substantially by the higher-loop or nonperturbative effects. Nevertheless, we adopt the one-loop formulas Eqs.(21) and (24) in the following sections, assuming optimistically that qualitative behavior can be obtained within one-loop formulas.

We are now ready to start the analysis of the gap equation. The running effect of the gauge coupling is taken into account by replacing the renormalization scale $(\mu)$ dependence with the gauge boson momentum $z$ :

$$
g_{D}^{2}(z)=\frac{\hat{g}^{2}(\mu=\sqrt{z})}{z^{\delta / 2}} .
$$

We start with the simplest case where the dimensionless gauge coupling is standing at the UV-FP $g_{*}^{2}:$

$$
g_{D}^{2}(z)=\frac{g_{*}^{2}}{z^{\delta / 2}}
$$

Plugging Eq. 28) into Eq. (16) we find that the $A \equiv 1$ gauge is given by a simple form

$$
\xi(z)=-\frac{(D-1)(D-4)}{D} .
$$

We note that Eq.(29) is merely a constant. The gauge fixing operator is thus a local one in this case at the UV-FP. 
It is straightforward to perform the angular integral in Eq.(18),

$$
K_{B}(x, y)=\frac{4(D-1)}{D} g_{*}^{2} \Omega_{\mathrm{NDA}}\left(\frac{1}{x^{D / 2-1}} \theta(x-y)+\frac{1}{y^{D / 2-1}} \theta(y-x)\right) .
$$

We thus obtain the gap equation

$$
B(x)=m_{0}+\frac{4(D-1)}{D} \kappa_{D} \int_{0}^{\Lambda^{2}} d y \frac{y^{D / 2-1} B(y)}{y+B^{2}(y)}\left(\frac{1}{x^{D / 2-1}} \theta(x-y)+\frac{1}{y^{D / 2-1}} \theta(y-x)\right),
$$

with

$$
\kappa_{D} \equiv C_{F} g_{*}^{2} \Omega_{\mathrm{NDA}} .
$$

We next try to solve the gap equation (31) analytically.

Differentiating Eq. (31) over $x$, we find that the integral equation (31) is equivalent to the differential equation

$$
\frac{d}{d x}\left[x^{D / 2} \frac{d}{d x} B\right]+\frac{2(D-1)(D-2)}{D} \kappa_{D} \frac{x^{D / 2-1} B}{x+B^{2}}=0,
$$

and a set (infrared and ultraviolet) of boundary conditions:

$$
\begin{aligned}
\left.x^{D / 2} \frac{d}{d x} B(x)\right|_{x=0}=0, & (\mathrm{IR}-\mathrm{BC}), \\
\left.\left(1+\frac{1}{2 \omega} x \frac{d}{d x}\right) B(x)\right|_{x=\Lambda^{2}} & =m_{0}, \quad(\mathrm{UV}-\mathrm{BC}),
\end{aligned}
$$

with $\omega$ being defined by ${ }^{3}$

$$
\omega \equiv \frac{1}{2}\left(\frac{D}{2}-1\right)
$$

The differential equation (33) is still non-linear and cannot be solved analytically. In Ref. [5], we discussed similar equation using a bifurcation method [27] in order to deal with the non-linearity. Here in this paper, we use a different method [28], in which Eq.(33) is replaced by a linearized one

$$
\frac{d}{d x}\left[x^{D / 2} \frac{d}{d x} B\right]+\frac{2(D-1)(D-2)}{D} \kappa_{D} \frac{x^{D / 2-1} B}{x+B_{0}^{2}}=0,
$$

combined with a subsidiary normalization condition

$$
B_{0} \equiv B(x=0) .
$$

The approximation Eq.(37) can be shown to work reasonably well in both high- and low-energy regions. It has also been used widely in the analysis of the dynamical chiral symmetry breaking.

It is now easy to solve the differential equation (37). Combining it with the IR-BC (34) and the normalization condition (38), we find that the solution is given in terms of the hypergeometric function,

$$
B(x)=B_{0} F\left(\omega(1+\tilde{\nu}), \omega(1-\tilde{\nu}), D / 2 ;-x / B_{0}^{2}\right), \quad \tilde{\nu} \equiv \sqrt{1-\kappa_{D} / \kappa_{D}^{\text {crit }}}
$$

\footnotetext{
3 The sign in the definition of $\omega$, Eq. 36, is opposite to the definition in Ref. [5].
} 
for $\kappa_{D}<\kappa_{D}^{\text {crit }}$, and

$$
B(x)=B_{0} F\left(\omega(1+i \nu), \omega(1-i \nu), D / 2 ;-x / B_{0}^{2}\right), \quad \nu \equiv \sqrt{\kappa_{D} / \kappa_{D}^{\text {crit }}-1}
$$

for $\kappa_{D}>\kappa_{D}^{\text {crit }}$, where $\kappa_{D}^{\text {crit }}$ is given by

$$
\kappa_{D}^{\mathrm{crit}}=\frac{D}{32} \frac{D-2}{D-1}
$$

The critical $\kappa_{D}^{\text {crit }}$ separates chiral symmetric and broken phases as shown below. The chiral symmetry breaking takes place for $\kappa_{D}>\kappa_{D}^{\text {crit }}$, while the theory remains chiral symmetric for $\kappa_{D}<\kappa_{D}^{\text {crit }}$. We also define the "critical coupling" $\hat{g}_{\text {crit }}$ for later purpose,

$$
\hat{g}_{\text {crit }}^{2} \equiv \frac{\kappa_{D}^{\text {crit }}}{C_{F} \Omega_{\mathrm{NDA}}}
$$

We note that the $\kappa_{D}^{\text {crit }}$ is larger than the previous calculation in the Landau gauge,

$$
\left.\kappa_{D}^{\text {crit }}=\frac{1}{8} \frac{D-2}{D-1}, \quad \text { (Landau gauge }\right)
$$

where the prescription Eq.(7) was adopted. The difference between Eq.(41) and Eq.(43) becomes significant for larger $D \gg 4$. Moreover, Eq.(11) indicates that the critical coupling is stronger than the NDA estimate $\kappa_{D}^{\text {crit }} \sim \mathcal{O}(1)$ for $D \gg 4$. This property can be related to the "bound state" problem in $D$ dimensional quantum mechanics. In order to investigate this issue, we will rewrite the gap equation into a form of equivalent Schrödinger-like equation in Appendix B.

We first consider the solution in the subcritical region, Eq.(39). In the asymptotic energy region $\left(x \gg B_{0}^{2}\right)$, Eq. (39) behaves as

$$
B(x)=B_{0}\left[\tilde{c}_{0}\left(\frac{x}{B_{0}^{2}}\right)^{-\omega(1-\tilde{\nu})}+\tilde{d}_{0}\left(\frac{x}{B_{0}^{2}}\right)^{-\omega(1+\tilde{\nu})}+\mathcal{O}\left(\left(\frac{x}{B_{0}^{2}}\right)^{-\omega(1-\tilde{\nu})-1}\right)\right],
$$

with $\tilde{c}_{0}$ and $\tilde{d}_{0}$ being given by

$$
\tilde{c}_{0} \equiv \frac{\Gamma(D / 2) \Gamma(2 \omega \tilde{\nu})}{\Gamma(\omega(1+\tilde{\nu})) \Gamma(1+\omega(1+\tilde{\nu}))}, \quad \tilde{d}_{0} \equiv \frac{\Gamma(D / 2) \Gamma(-2 \omega \tilde{\nu})}{\Gamma(\omega(1-\tilde{\nu})) \Gamma(1+\omega(1-\tilde{\nu}))} .
$$

Plugging Eq. (44) into the UV-BC Eq. 35, we obtain

$$
\frac{1}{2}(1+\tilde{\nu}) \tilde{c}_{0} B_{0}\left(\frac{\Lambda^{2}}{B_{0}^{2}}\right)^{-\omega(1-\tilde{\nu})} \simeq m_{0}
$$

The non-trivial solution $B_{0} \neq 0$ exists only when $m_{0} \neq 0$, i.e., the dynamical chiral symmetry breaking does not occur in the subcritical region $\kappa_{D}<\kappa_{D}^{\text {crit }}$.

The situation differs significantly for $\kappa_{D}>\kappa_{D}^{\text {crit }}$. The high energy behavior of Eq. (40) is

$$
B(x)=B_{0}\left[c_{0}\left(\frac{x}{B_{0}^{2}}\right)^{-\omega(1-i \nu)}+d_{0}\left(\frac{x}{B_{0}^{2}}\right)^{-\omega(1+i \nu)}+\mathcal{O}\left(\left(\frac{x}{B_{0}^{2}}\right)^{-\omega-1}\right)\right],
$$

with

$$
c_{0} \equiv \frac{\Gamma(D / 2) \Gamma(2 i \omega \nu)}{\Gamma(\omega(1+i \nu)) \Gamma(1+\omega(1+i \nu))}, \quad d_{0} \equiv c_{0}^{*}
$$


Unlike the solution at $\kappa_{D}<\kappa_{D}^{\text {crit }}$, Eq. (46) is an oscillating function. Actually, Eq.(46) can be written as

$$
B(x) \simeq 2\left|c_{0}\right| B_{0}\left(\frac{x}{B_{0}^{2}}\right)^{-\omega} \sin \left[\theta+\omega \nu \ln \frac{x}{B_{0}^{2}}\right],
$$

with $\theta$ being given by $e^{2 i \theta}=-c_{0} / d_{0}$. The UV-BC Eq.(35) thus reads

$$
\sqrt{1+\nu^{2}}\left|c_{0}\right| B_{0}\left(\frac{\Lambda^{2}}{B_{0}^{2}}\right)^{-\omega} \sin \left[\theta+\omega \nu \ln \frac{\Lambda^{2}}{B_{0}^{2}}+\tan ^{-1} \nu\right]=m_{0} .
$$

Non-trivial solutions $B_{0} \neq 0$ exist even in the chiral limit $m_{0}=0$ of Eq.(48):

$$
B_{0} \simeq C \Lambda \exp \left[-\frac{n \pi}{(D / 2-1) \nu}\right],
$$

with $n$ being a positive integer describing the number of nodes in $B(x)$. The $n=1$ solution corresponds to the nodeless one. Here the factor $C$ is given by $C \equiv \exp \left(\left(\theta+\tan ^{-1} \nu\right) /((D / 2-1) \nu)\right)$, which remains finite in the $\nu \rightarrow 0$ limit $\left(\nu \equiv \sqrt{\kappa_{D} / \kappa_{D}^{\text {crit }}-1}\right)$.

Although we found infinite number of solutions in Eq.(49) labeled by $n$, these solutions may correspond unstable vacua. We need to evaluate the vacuum energy in order to find the true vacuum with minimum energy. We thus compare energies of different vacua $(n=1,2, \cdots)$ by using the CornwallJackiw-Tomboulis (CJT) effective potential [23]. Within the improved ladder approximation, the CJT potential is given by

$$
\begin{aligned}
-\frac{V_{\mathrm{CJT}}\left[B, m_{0}, \Lambda\right)}{\eta N N_{f} \Omega_{\mathrm{NDA}}}= & \int_{0}^{\Lambda^{2}} d x x^{D / 2-1}\left\{\frac{1}{2} \ln \left(1+\frac{B^{2}(x)}{x}\right)-\frac{B^{2}(x)-m_{0} B(x)}{x+B^{2}(x)}\right\} \\
& +\frac{1}{2} C_{F} \int_{0}^{\Lambda^{2}} d x x^{D / 2-1} \frac{B(x)}{x+B^{2}(x)} \int_{0}^{\Lambda^{2}} d y y^{D / 2-1} \frac{B(y)}{x+B^{2}(y)} K_{B}(x, y) .
\end{aligned}
$$

It is easy to show that the stationary condition of the CJT potential $\delta V_{\mathrm{CJT}} / \delta B=0$ is identical to the gap equation Eq.(17).

Using a scaling technique described in Ref. 29] we can evaluate the CJT effective potential,

$$
-\frac{D}{\eta N N_{f} \Omega_{\mathrm{NDA}}} V_{\mathrm{CJT}}\left[B_{\mathrm{sol}}, m_{0}, \Lambda\right)=\Lambda^{D} \ln \left(1+\frac{B_{\Lambda}^{2}}{\Lambda^{2}}\right)+\frac{D}{4(D-1) \kappa_{D}} \Lambda^{D-2}\left(B_{\Lambda}-m_{0}\right) m_{0},
$$

where

$$
B_{\Lambda} \equiv B_{\text {sol }}\left(x=\Lambda^{2}\right),
$$

with $B_{\text {sol }}$ being the solution of the gap equation Eq.(31). We note that the vacuum energy $V_{\text {CJT }}$ is a decreasing function of $\left|B_{\Lambda}\right|$ in the chiral limit $m_{0}=0$. Combining Eq.(49) and Eq.(47), it is easy to obtain

$$
\left|B_{\Lambda}\right| \simeq \frac{2\left|c_{0}\right| \nu}{\sqrt{1+\nu^{2}}} \Lambda\left(\frac{B_{0}}{\Lambda}\right)^{D / 2}
$$

We thus find that the vacuum energy is a decreasing function of $B_{0}$. It is now straightforward to show that the vacuum with minimum energy in the chiral limit $m_{0}=0$ corresponds to the $n=1$ solution (largest $\left.B_{0}\right)^{4}$ in Eq.(49) at the super-critical $\kappa_{D}>\kappa_{D}^{\text {crit }}$.

4 It should be mentioned that the $n=1$ solution is not an absolute minimum, but a saddle point of the CJT potential. However, this fact does not indicate the instability of this vacuum, since negative curvature in the CJT potential does not necessarily imply the existence of a tachyonic mode. We will discuss another potential with possibly better perspective in Appendix A. 
We thus obtain the scaling relation for $\kappa_{D} \simeq \kappa_{D}^{\mathrm{crit}}$,

$$
B_{0} \propto \Lambda \exp \left[\frac{-\pi}{(D / 2-1) \sqrt{\kappa_{D} / \kappa_{D}^{\text {crit }}-1}}\right] .
$$

As pointed out in Ref. [5], the chiral phase transition Eq. (54) is an essential-singularity type (as a result of "conformal phase transition" 16]), which enables us to obtain a hierarchy between the cutoff $\Lambda$ and the dynamical mass $B_{0}$ in a model with $\kappa_{D}$ sufficiently close to the critical $\kappa_{D}^{\text {crit }}$. Actually, it is easy to realize a hierarchy of $\mathcal{O}(10)$ level without any fine-tuning, e.g., $\Lambda / B_{0} \simeq 12$ in the $S U(3)$ gauge theory with $N_{f}=5$ and $D=4+2$.

Note, however, that $\kappa_{D}$ is not an adjustable free parameter, but a definite number once the model is set up. We are thus not able to obtain arbitrarily large hierarchy $\Lambda / B_{0}$ in models standing at the UV-FP. In order to clarify the point, it is useful to translate $\kappa_{D}^{\text {crit }}$ to critical number of flavors $N_{f}^{\text {crit }}$ by noting that $\kappa_{D}$ depends on $N_{f}$,

$$
N_{f}^{\text {crit }}=\frac{3}{\eta T_{R}}\left[\frac{26-D}{6} C_{G}-\frac{C_{F}}{\frac{2}{D-4}+1} \frac{32(D-1)}{D(D-2)}\right],
$$

where we have used Eq.(22) and Eq.(24). ( $\kappa_{D}<\kappa_{D}^{\text {crit }}$ corresponds to $N_{f}<N_{f}^{\text {crit }}$.) For $S U(3)$ gauge theories, the critical $N_{f}$ is evaluated as,

$$
N_{f}^{\text {crit }} \simeq \begin{cases}4.2, & \text { for } D=4+2 \\ 1.8, & \text { for } D=4+4 \\ 0.8, & \text { for } D=4+6\end{cases}
$$

The largest hierarchy in $S U(3)$ gauge theories is then obtained when $N_{f}=5, N_{f}=2$ and $N_{f}=1$ in $D=4+2, D=4+4$ and $D=4+6$ dimensions, respectively.

In order to estimate the uncertainties coming from the compactification scale $R^{-1}$, we next consider the effects of an infrared (IR) cutoff $M_{0}^{2} \sim R^{-2}$. It is shown in Appendix $\mathrm{C}$ that the critical $\kappa_{D}$ is a function of $M_{0}^{2}$ under the presence of IR cutoff $M_{0}^{2}$,

$$
\kappa_{D}^{\mathrm{crit}}\left(M_{0}^{2}\right)=\kappa_{D}^{\mathrm{crit}}\left(M_{0}^{2}=0\right)\left(1+\nu_{c}^{2}\left(M_{0}^{2}\right)\right)
$$

with $\kappa_{D}^{\text {crit }}\left(M_{0}^{2}=0\right)$ being the critical point without IR cutoff and given by Eq.(41). $\nu_{c}\left(M_{0}^{2}\right)$ is a solution of

$$
2 \tan ^{-1} \nu_{c}+\omega \nu_{c} \ln \frac{\Lambda^{2}}{M_{0}^{2}}=\pi
$$

For $\Lambda \gg M_{0}, \nu_{c}\left(M_{0}^{2}\right)$ is thus given by

$$
\nu_{c}\left(M_{0}^{2}\right) \simeq \frac{\pi}{2+\omega \ln \frac{\Lambda^{2}}{M_{0}^{2}}} \ll 1
$$

The difference between $\kappa_{D}^{\text {crit }}\left(M_{0}^{2} \neq 0\right)$ and $\kappa_{D}^{\text {crit }}\left(M_{0}^{2}=0\right)$ can be neglected for sufficiently large UV cutoff $\Lambda$.

On the other hand, the scaling behavior of $B\left(M_{0}^{2}\right)$ coincides with Eq.(54) (see also Eq. (49)) for $M_{0} \ll B\left(M_{0}^{2}\right) \ll \Lambda$, while

$$
B\left(M_{0}^{2}\right) \propto M_{0} \frac{\sqrt{\nu-\nu_{c}\left(M_{0}^{2}\right)}}{\nu_{c}\left(M_{0}^{2}\right)}
$$




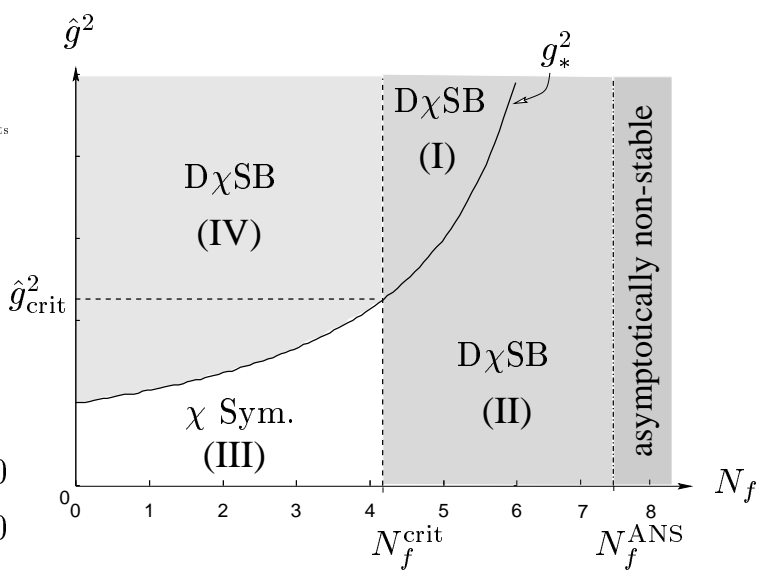

FIG. 2: The phase diagram of $S U(3)$ gauge theory in $D=4+2$ dimensions. While the theory remains chiral symmetric for $N_{f}<N_{f}^{\text {crit }}$ with $\hat{g}<g_{*}$, the dynamical chiral symmetry breaking takes place in the entire region of $\hat{g}$ with a sufficiently large cutoff $\Lambda$ for $N_{f}^{\text {crit }}<N_{f}<N_{f}^{\text {ANS }}=7.5$. The theory becomes asymptotically non-stable without UV-FP for $N_{f}>N_{f}^{\mathrm{ANS}}$.

for $B\left(M_{0}^{2}\right) \ll M_{0}$. Eq.(60) indicates mean-field type scaling. We thus need a fine-tuning of model in order to realize hierarchy between the fermion mass $B\left(M_{0}^{2}\right)$ and the IR cutoff $M_{0}\left(\sim R^{-1}\right)$.

If the effects of the compactification scale $R^{-1}$ can be mimicked by the IR cutoff $M_{0}$, the result Eq.(60) implies that the dynamical fermion mass $B_{0}$ cannot be made extremely smaller than $R^{-1}$ without severe fine-tuning. It is, however, relatively easy to achieve $\mathcal{O}(10)$ level hierarchy between UV cutoff $\Lambda$ and $B_{0}$ as we have discussed before.

\section{PHASE STRUCTURE}

We have so far discussed the chiral phase transition assuming that the gauge coupling $\hat{g}$ is standing at the UV-FP. We found the critical $N_{f}^{\text {crit }}$, Eq.(55). The chiral symmetry breaking takes place only when $N_{f}>N_{f}^{\text {crit }}$ at the UV-FP.

In particle models with extra dimensions, however, the gauge coupling $\hat{g}$ is not necessarily on its UV-FP, $g_{*}$. The bulk QCD coupling of the ACDH scenario of the top condensate, for example, is shown to be below its UV-FP. In this section, we therefore try to draw more concrete picture of the phase diagram including the gauge coupling strength apart from the UV-FP. It is illuminating to discuss the phase diagram in the $\hat{g}^{2}-N_{f}$ plane.

In the following analysis, particular interests are paid for $S U(3)$ gauge theories in $D=4+2$ dimensions, in which the critical $N_{f}$ is evaluated as $N_{f}^{\text {crit }} \simeq 4.2$. Since the chiral symmetry breaking is our main concern, we take the chiral limit $m_{0}=0$ in this section.

Before starting the detailed numerical analysis, we first discuss the qualitative picture of the phase diagram (Fig.2) by using the result of the gap equation at the UV-FP.

Let us start with the case $N_{f}^{\text {ANS }}>N_{f}>N_{f}^{\text {crit }}$. The typical behavior of the beta function of the dimensionless gauge coupling $\hat{g}$ is depicted in Fig. 3 with this $N_{f}$. The UV-FP is above the critical coupling $\hat{g}_{\text {crit }}$. The bulk fermion then acquires its dynamical mass proportional to the cutoff $\Lambda$, Eq.(54), even at the UV-FP. We therefore expect the chiral symmetry breaking takes place in the strongly coupled regime $\hat{g}>g_{*}$ (region I in Fig. 2). Since the gauge coupling $\hat{g}$ approaches quickly to its UV-FP in the asymptotic region, the coupling exceeds its critical value for sufficiently large energy scale even in the weakly coupled region (region II). It is then expected that dynamical chiral symmetry breaking occurs even in region II for sufficiently large cutoff. In order to keep the fermion 
mass finite, the cutoff $\Lambda$ needs to be finite.

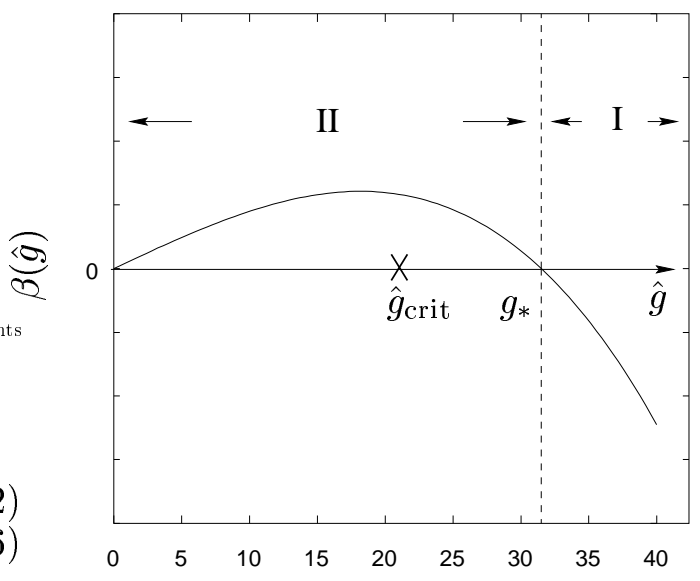

FIG. 3: The beta function of $S U(3)$ gauge theory with $N_{f}=6$ in $D=4+2$ dimensions. The UV-FP is above the critical coupling $\hat{g}_{\text {crit }}$.

The situation should be substantially different for $N_{f}<N_{f}^{\text {crit }}$. (See Fig. 田) In this case, the fermion remains massless at the UV-FP $\hat{g}=g_{*}$ or $\Lambda \frac{(D)}{\mathrm{MS}}=0$ with $\Lambda \frac{(D)}{\mathrm{MS}}$ being the scale of the gauge theory defined in Eq.(26). The gauge coupling $\hat{g}$, therefore, does not exceeds its critical value $\hat{g}_{\text {crit }}$ in the weakly coupled regime $\left(\Lambda \frac{(D)}{\mathrm{MS}}\right)^{2}<0$ (region III). On the other hand, the gauge coupling in the strongly coupled phase $\left(\Lambda \frac{(D)}{\mathrm{MS}}\right)^{2}>0$ (region IV) becomes extremely strong at the infrared region $\mu^{2} \simeq\left(\Lambda_{\mathrm{MS}}\right)^{2}$ and the coupling becomes stronger than its critical value $\hat{g}_{\text {crit }}$. It is therefore expected that the fermion acquires its dynamical mass $M^{2} \sim\left(\Lambda \frac{(D)}{\mathrm{MS}}\right)^{2}$ in region IV, while the theory remains chiral symmetric in region III. The cutoff $\Lambda$ can be arbitrarily large in the analysis of the gap equation for $N_{f}<N_{f}^{\text {crit }}$.

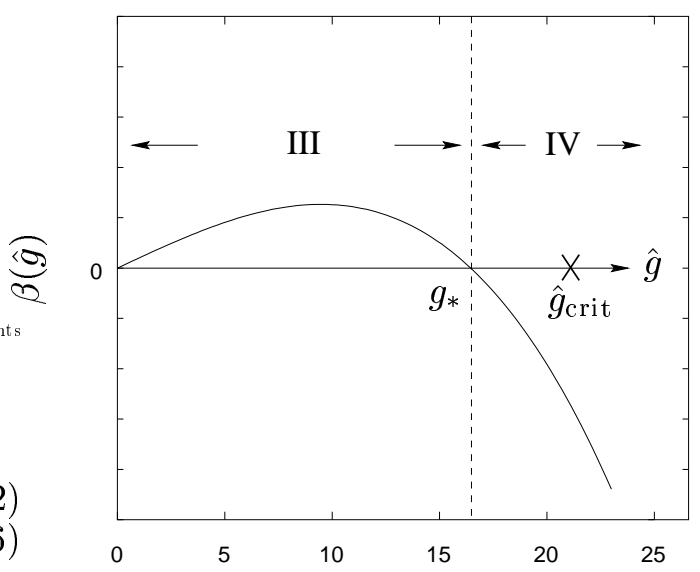

FIG. 4: The beta function of $S U(3)$ gauge theory with $N_{f}=2$ in $D=4+2$ dimensions. The UV-FP is below the critical coupling $\hat{g}_{\text {crit }}$.

If we neglect effects of the weak gauge interactions, the minimal ACDH scenario corresponds to the $D=4+2$ dimensional $S U(3)$ (QCD) gauge theory with $N_{f}=2$ and $\hat{g}<g_{*}$. Eq.(56) implies 
$N_{f}^{\text {crit }} \simeq 4.2$ in this case and Fig. 2 shows the model is in its chiral symmetric phase (region III). The QCD gauge coupling is thus not strong enough to trigger the chiral condensate in this scenario no matter how large the cutoff $\Lambda$ is. The model thus needs to be modified in order to explain the electroweak symmetry breaking. For example, the $D=4+4$ dimensional version of the ACDH scenario is shown to be in the chiral symmetry breaking phase (region II) $\left(N_{f}^{\text {crit }} \simeq 1.8\right)$ and it may explain the mass of weak gauge bosons.

It is also worth pointing out that the region IV in the phase diagram (Fig. 2) may open an interesting possibility in the model buildings of the electroweak symmetry breaking. The region IV is very interesting because we can formally take $\Lambda \rightarrow \infty$ limit in the analysis of the gap equation. The low energy predictions of the models in this phase are thus insensitive to the physics around the UV cutoff. One of the possibilities is an idea of "bulk technicolor" model. The phenomenology of this scenario will be discussed in a separated publication.

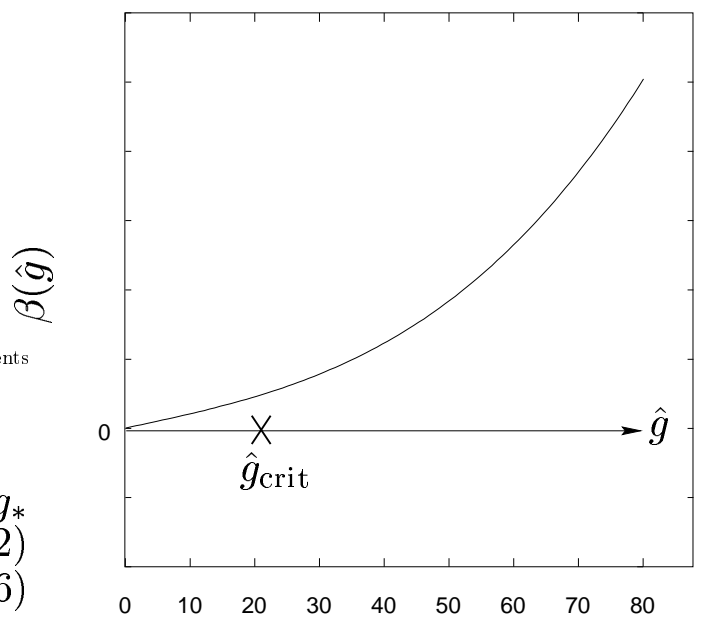

FIG. 5: The beta function of $S U(3)$ gauge theory with $N_{f}=8$ in $D=4+2$ dimensions. The theory becomes asymptotically non-stable (ANS).

Finally, we consider the case with $N_{f}>N_{f}^{\text {ANS }}$, where the theory becomes asymptotically nonstable. (See Fig. E). The gauge coupling $\hat{g}$ grows without a bound and exceeds $\hat{g}_{\text {crit }}$ in the high energy region. The chiral symmetry is then expected to be broken spontaneously for sufficiently large cutoff $\Lambda$.

In order to confirm these expectations, however, we need to investigate the gap equation with the gauge coupling strength away from its fixed point, which we will perform in the following subsections.

\section{A. $\quad N_{f}<N_{f}^{\text {crit }}$}

Let us first consider the chiral symmetry breaking in the strongly interacting phase $\left(\Lambda \frac{(D)}{\mathrm{MS}}\right)^{2}>0$ with $N_{f}<N_{f}^{\text {crit }}($ region IV).

Note here that Eq. (26) has a difficulty associated with the (tachyonic) pole singularity for $\left(\Lambda \frac{(D)}{\mathrm{MS}}\right)^{2}>$ 0 (strongly coupled phase). Such a singularity appears only when the gauge coupling becomes extremely strong. So it should be an artifact of one-loop approximation of the beta function. In order to avoid the difficulty, we make an ansatz of the dimensionless gauge coupling $\hat{g}^{2} \equiv \mu^{2} g_{D}^{2}$ in the infrared 
region $\mu^{2}<\tau\left(\Lambda \frac{(D)}{\mathrm{MS}}\right)^{2}$ with $\tau>1$

$$
\hat{g}^{2}\left(\mu^{2}\right) \Omega_{\mathrm{NDA}}=\frac{1}{-2 b^{\prime}} \frac{1}{(\tau-1)^{2}}\left[\tau^{2}-\frac{\mu^{2}}{\left(\Lambda \frac{(D)}{\mathrm{MS}}\right)^{2}}\right],
$$

for $\mu^{2}<\tau\left(\Lambda \frac{(D)}{\mathrm{MS}}\right)^{2}$. The form Eq. (61) is a linear function of $\mu^{2}$ and is taken so as to make $\hat{g}^{2}\left(\mu^{2}\right)$ and its first derivative continuous at $\mu^{2}=\tau\left(\Lambda \frac{(D)}{\mathrm{MS}}\right)^{2}$. The regulator $\tau\left(\Lambda \frac{(D)}{\mathrm{MS}}\right)^{2}$ is chosen to make the coupling at $g_{D}^{2}\left(\mu^{2}=0\right)$ sufficiently large:

$$
\begin{aligned}
\hat{g}_{\mathrm{IR}}^{2} \Omega_{\mathrm{NDA}} & =\left.\hat{g}^{2}\left(\mu^{2}\right) \Omega_{\mathrm{NDA}}\right|_{\mu^{2}=0} \\
& =\frac{1}{-2 b^{\prime}} \frac{\tau^{2}}{(\tau-1)^{2}} \sim \mathcal{O}(1) .
\end{aligned}
$$

The behaviors of the gauge coupling of $D=4+2 S U(3) N_{f}=2$ gauge theory are shown in Fig. 6 for positive $\left(\Lambda \frac{(D)}{\mathrm{MS}}\right)^{2}=10^{2} M_{0}^{2}>0$. Each lines correspond to the non-reguralized, and the reguralized $\hat{g}$ with $\hat{g}_{\mathrm{IR}} \Omega_{\mathrm{NDA}}=0.2$ and 0.8 . The non-reguralized $\hat{g}$ diverges at the scale $\left(\Lambda \frac{(D)}{\mathrm{MS}}\right)^{2}$. Corresponding behaviors of the non-local gauge fixing parameter $\xi$ are shown in Fig. 7 .

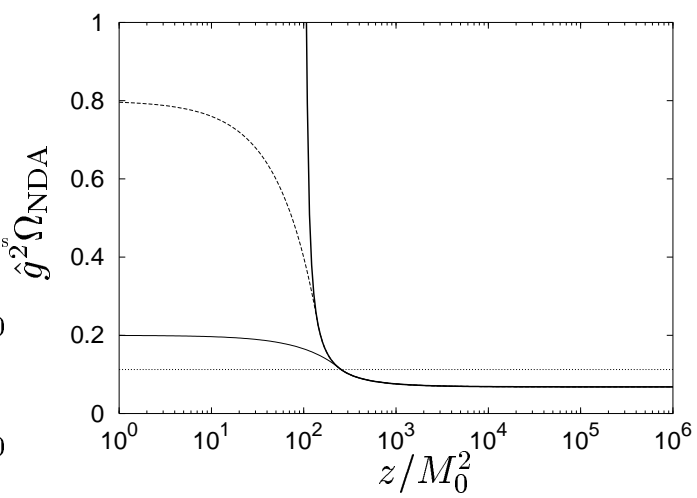

FIG. 6: The momentum dependence of the running gauge coupling in the strongly coupled phase of the $D=4+2 S U(3)$ gauge theory $\left(N_{f}=2,\left(\Lambda_{\overline{\mathrm{MS}}}^{(D)}\right)^{2}=10^{2} M_{0}^{2}\right)$. The infrared regularization $g_{\mathrm{IR}}$ is taken to be 0.2 (solid line) and 0.8 (dashed line). The dotted line is the critical coupling $\left(\hat{g}_{\mathrm{crit}}^{2} \Omega_{\mathrm{NDA}}=9 / 80\right)$.

The gap equation is solved in a numerical manner by adopting the recursion method [5]. We also introduce an infrared cutoff $M_{0}$. In the following numerical analysis, $\left(\Lambda_{\overline{\mathrm{MS}}}^{(D)}\right)^{2}=10^{2} M_{0}^{2}$ and $\Lambda^{2}=10^{6} M_{0}^{2}$ are assumed.

The numerical solution of the gap equation $B(x) / M_{0}$ is shown in Fig. 8 for various choice of the infrared regulator $\hat{g}_{\mathrm{IR}}^{2} \Omega_{\mathrm{NDA}}$. We find

$$
B\left(M_{0}^{2}\right) \sim \Lambda \frac{(D)}{\mathrm{MS}}=\Lambda \sqrt{1-\frac{g_{*}^{2}}{\hat{g}^{2}(\mu=\Lambda)}}
$$

for sufficiently large $\hat{g}_{\mathrm{IR}}$ as we have expected before. The solution is insensitive to the choice of the UV cutoff $\Lambda^{2}$. We can thus formally define a continuum limit (infinite cutoff limit), which implies that the low-energy physics becomes insensitive to the details of the physics around cutoff. It should be emphasized that this phase may be useful within the framework of local field theory, no matter whatever physics may exist behind the UV cutoff. 


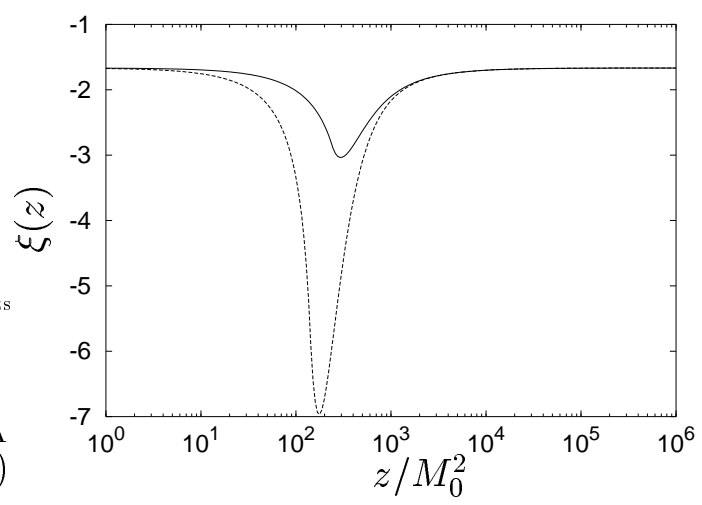

FIG. 7: The momentum dependence of the nonlocal gauge fixing parameter in the strongly coupled phase of the $D=4+2 S U(3)$ gauge theory $\left(N_{f}=2,\left(\Lambda \frac{(D)}{\mathrm{MS}}\right)^{2}=10^{2} M_{0}^{2}\right)$. The infrared regularization $g_{\mathrm{IR}}$ is taken to be 0.2 (solid line) and 0.8 (dashed line).

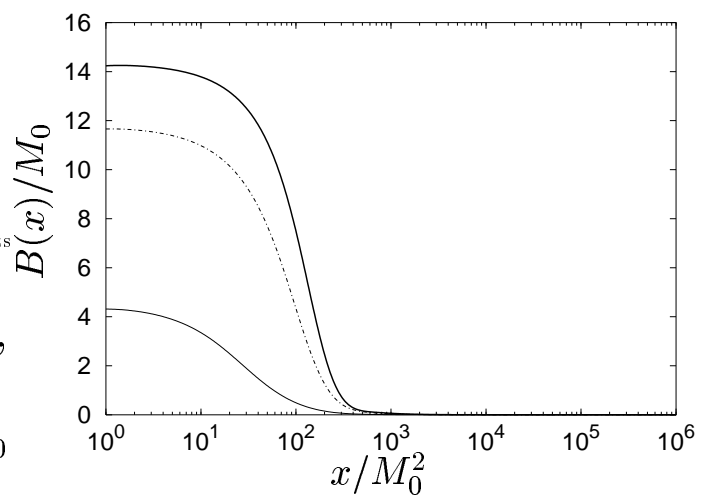

FIG. 8: The solution of the gap equation of $D=4+2$ dimensional $S U(3)$ gauge theory with $N_{f}=2$. $\left(\Lambda \frac{(D)}{\mathrm{MS}}\right)^{2}=10^{2} M_{0}^{2}$ and $\Lambda^{2}=10^{6} M_{0}^{2}$ are assumed. The results with various choice of the infrared regulator $\hat{g}_{\mathrm{IR}}^{2} \Omega_{\mathrm{NDA}}=0.2$ (solid line), 0.5 (dashed line), 0.8 (bold line) are depicted.

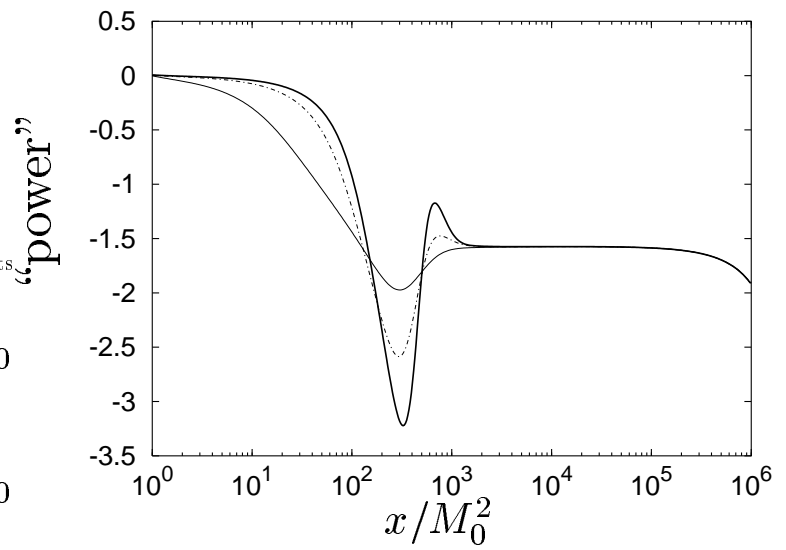

FIG. 9: The "power" behavior of the numerical solution. The infrared regulator is taken as $\hat{g}_{\mathrm{IR}}^{2} \Omega_{\mathrm{NDA}}=0.2$ (solid line), 0.5 (dashed line), 0.8 (bold line). 
We next consider the high-energy $\left(x \gg\left(\Lambda \frac{(D)}{\mathrm{MS}}\right)^{2} \sim B^{2}\right)$ behavior of the mass function. In the asymptotic energy region, the gauge coupling strength approaches very quickly to its UV-FP. We therefore expect that the asymptotic solution satisfies the differential equation Eq.(33) and the UVBC Eq.(35) which were derived originally at the UV-FP. On the other hand, the infrared behavior of the solution should be substantially different from the solution at the UV-FP. We therefore do not adopt the IR-BC Eq.(34).

Eq.(33) can be approximated further for $x \gg B^{2}$,

$$
\begin{aligned}
\frac{d}{d x} & {\left[x^{D / 2} \frac{d}{d x} B\right] } \\
& +\frac{2(D-1)(D-2)}{D} \kappa_{D} x^{D / 2-2} B=0 .
\end{aligned}
$$

It is easy to solve Eq.(64) and Eq.(35). We find the asymptotic solution is given by

$$
B(x) \propto\left(\frac{x}{\Lambda^{2}}\right)^{-\omega(1+\tilde{\nu})}-\frac{1-\tilde{\nu}}{1+\tilde{\nu}}\left(\frac{x}{\Lambda^{2}}\right)^{-\omega(1-\tilde{\nu})},
$$

with $\tilde{\nu} \equiv \sqrt{1-\kappa_{D} / \kappa_{D}^{\text {crit }}}=\sqrt{1-g_{*}^{2} / \hat{g}_{\text {crit }}^{2}}$. The second term is negligible for $\Lambda^{2} \gg x$. We thus expect that the mass function $B$ behaves as

$$
B(x) \propto\left(\frac{x}{\Lambda^{2}}\right)^{-\omega(1+\tilde{\nu})}
$$

in the energy region $\Lambda^{2} \gg x \gg\left(\Lambda \frac{(D)}{\mathrm{MS}}\right)^{2}$ if a non-trivial $B \neq 0$ solution exists.

In order to confirm the above expectation, we next plot the "power" behavior of the numerical solution of the mass function

$$
\text { "power" }=\frac{x}{B(x)} \frac{d B(x)}{d x}
$$

in Fig.9. The asymptotic behavior ${ }^{5}$ Eq. (66) is consistent with "power" $\simeq-1.6$ in Fig.9, which agrees well with the expected value $-\omega(1+\tilde{\nu})$ where $\omega=1, \tilde{\nu} \simeq 0.6$ for the $D=4+2$ dimensional $S U(3)$ gauge theory with $N_{f}=2$.

We also note that the "power" is related to the anomalous dimension of the fermion mass $\gamma_{m}$ as "power" $=\gamma_{m} / 2-(D / 2-1)$ [5]. The anomalous dimension $\gamma_{m}$ in the asymptotic region is then given by

$$
\gamma_{m}=\left(\frac{D}{2}-1\right)\left(1-\sqrt{1-g_{*}^{2} / \hat{g}_{\text {crit }}^{2}}\right) .
$$

We next examine the absolute magnitude of the mass function in the asymptotic region. For such a purpose, we show a log-log plot of the mass function in the asymptotic region. (See Fig.10. ) We find that the asymptotic mass function becomes insensitive to the infrared regulator $\hat{g}_{\mathrm{IR}}$ if we take $\hat{g}_{\mathrm{IR}}^{2} \Omega_{\mathrm{NDA}} \gtrsim 0.5$.

The infrared behavior of the solution depends significantly on the choice of the infrared regulator $\hat{g}_{\mathrm{IR}}^{2}$. The infrared behavior is therefore not enough trustworthy in this calculation. It should be emphasized, however, that the ultraviolet behavior is relatively insensitive to the choice of infrared regulator.

5 The behavior near the cutoff $x \simeq \Lambda^{2}$ in Fig. 1 is an artifact due to the sharp cutoff introduced in the analysis of the gap equation [30]. 


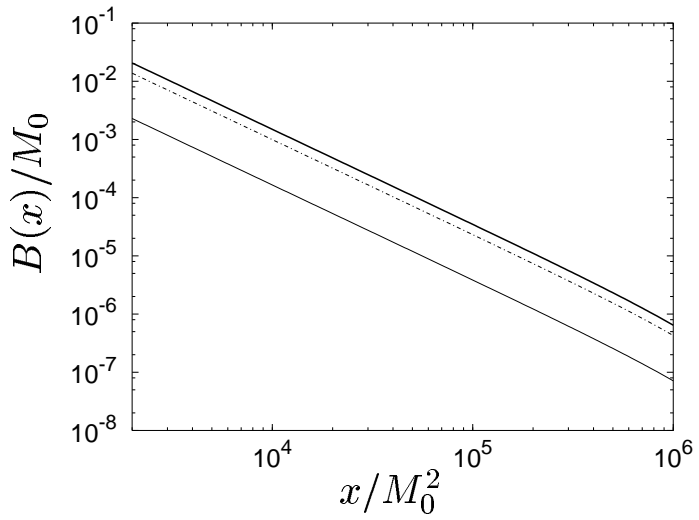

FIG. 10: The asymptotic behavior of the mass function for $\hat{g}_{I R}^{2} \Omega_{\mathrm{NDA}}=0.2$ (solid line), 0.5 (dashed line), 0.8 (bold line).

We have so far discussed the case where $\Lambda \frac{(D)}{\mathrm{MS}}$ is sufficiently large compared with the IR cutoff $M_{0}$ and found the dynamical chiral symmetry breaking $B\left(M_{0}^{2}\right) \sim \Lambda \frac{(D)}{\mathrm{MS}}$. The situation differs substantially for $\Lambda \frac{(D)}{\mathrm{MS}} \ll M_{0}$, where the gauge coupling $\hat{g}$ cannot exceeds its critical value of the chiral phase transition $\hat{g}_{\text {crit }}$. Actually, the dynamical chiral symmetry breaking does not take place for $\Lambda_{\frac{(D)}{\mathrm{MS}}} \ll M_{0}$.

Similar analysis is also performed in the weakly interacting phase $\left(\Lambda_{\overline{\mathrm{MS}}}^{(D)}\right)^{2}<0$ (region III). As we expected before, we find no signal of chiral symmetry breaking in this phase with $N_{f}<N_{f}^{\text {crit }}$.

\section{B. $\quad N_{f}>N_{f}^{\text {crit }}$}

We next discuss gauge theories with $N_{f}>N_{f}^{\text {crit }}$ (regions I and II). In these models, the UV-FP of the gauge coupling is strong enough to trigger the dynamical chiral symmetry breaking. We therefore expect that the bulk fermion acquires its dynamical mass even in the weakly coupled phase $\left(\Lambda_{\overline{M S}}\right)^{2}<0$ (region II). In order to confirm this expectation, we next investigate the gap equation with negative $\left(\Lambda \frac{(D)}{\mathrm{MS}}\right)^{2}$, i.e., weakly coupled phase with $N_{f}>N_{f}^{\text {crit }}$.

Fig. 11 shows the behavior of the $\overline{\mathrm{MS}}$ gauge coupling of $S U(3)$ gauge theory with $D=4+2, N_{f}=6$. The scale $\left(\Lambda \frac{(D)}{\mathrm{MS}}\right)^{2}$ is taken as $\left(\Lambda \frac{(D)}{\mathrm{MS}}\right)^{2} / M_{0}^{2}=-100$ in this figure. The gauge coupling approaches its UV-FP $g_{*}^{2} \Omega_{\mathrm{NDA}}=0.25$ very quickly. We expect that the dynamical chiral symmetry breaking occurs when the gauge coupling strength exceeds its critical value:

$$
C_{F} \hat{g}^{2} \Omega_{\mathrm{NDA}}>\kappa_{D}^{\mathrm{crit}}=\frac{3}{20},
$$

which is actually satisfied for $\mu^{2} \gtrsim\left|\left(\Lambda \frac{(D)}{\mathrm{MS}}\right)^{2}\right|$.

It is straightforward to solve the gap equation numerically. We find that the dynamical chiral symmetry breaking actually occurs when the cutoff $\Lambda$ is large enough, $\Lambda^{2} \gtrsim 5\left|\left(\Lambda_{\overline{M S}}^{(D)}\right)^{2}\right|$, with above mentioned parameters. Fig.12 shows the scaling behavior of $B\left(M_{0}^{2}\right) / \Lambda$ as a function of $\Lambda^{2} /\left|\left(\Lambda_{\overline{M S}}^{(D)}\right)^{2}\right|$. It should be emphasized that the cutoff $\Lambda$ can be determined in this case once $B\left(M_{0}^{2}\right)$ and $\left(\Lambda_{\overline{\mathrm{MS}}}^{(D)}\right)^{2}$ are fixed.

The top-mode standard model (TMSM) with extra dimensions would be one of the most important applications of this phase. As we have discussed before, we note that the cutoff $\Lambda^{2}$ is, in principle, 


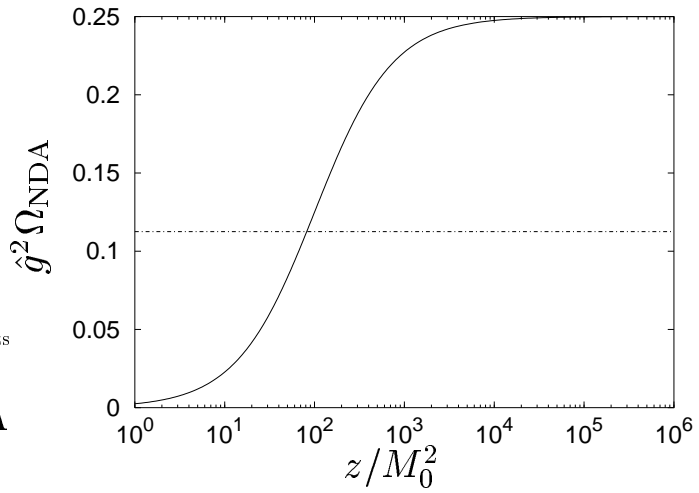

FIG. 11: The running of gauge coupling strength. $D=4+2, N=3, N_{f}=6,\left(\Lambda \frac{(D)}{\mathrm{MS}}\right)^{2} / M_{0}^{2}=-100$. The dashed line is the critical coupling $\left(\hat{g}_{\text {crit }}^{2} \Omega_{\mathrm{NDA}}=9 / 80\right)$.

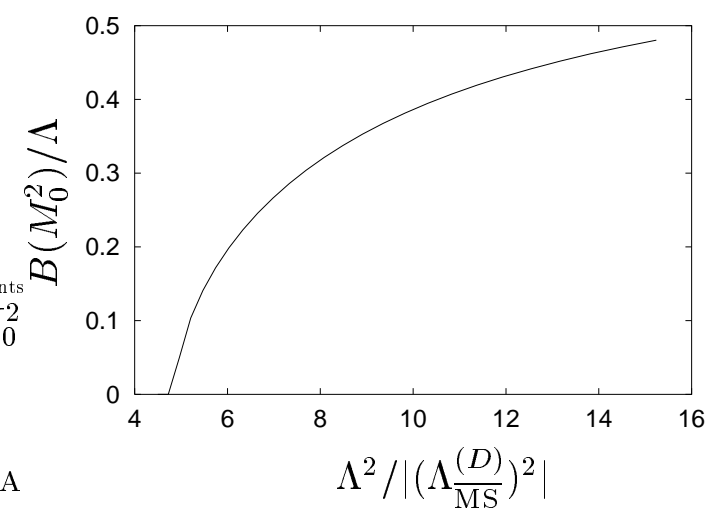

FIG. 12: The scaling behavior of $B\left(M_{0}^{2}\right) / \Lambda$ as a function of $\Lambda^{2} /\left|\left(\Lambda_{\overline{\mathrm{MS}}}^{(D)}\right)^{2}\right|$ in the $D=4+2$ dimensional $S U(3)$ theory with $N_{f}=6$. $\left(\Lambda \frac{(D)}{\mathrm{MS}}\right)^{2} / M_{0}^{2}=-100$ is assumed.

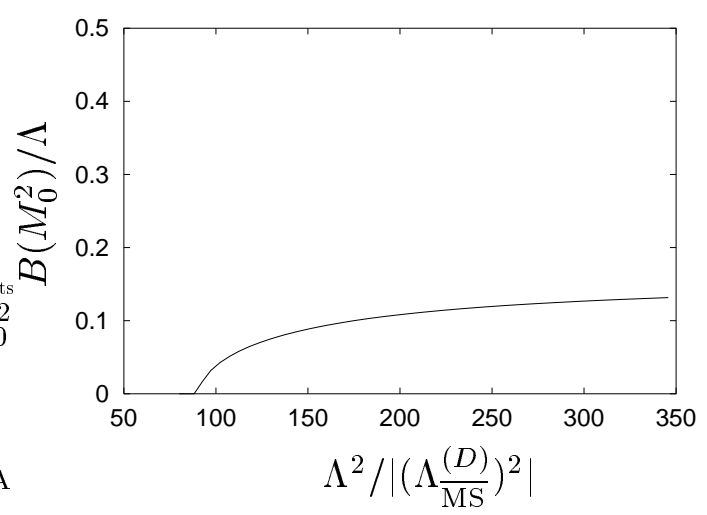

FIG. 13: The scaling behavior with $N_{f}=5$. Other parameters are the same as Fig. 12 . 
a calculable parameter in the analysis of the gap equation. Once the cutoff $\Lambda$ is determined, we can evaluate the decay constant of the NG boson (VEV of Higgs) $v$ by using the Pagels-Stokar formula [31. ${ }^{6}$ We are thus able to test the scenario by comparing the calculated $v$ with the actual value $v \simeq 250 \mathrm{GeV}$. In other words, we can "predict" the UV cutoff once we fix the VEV $v$ to the actual value. This property is due to the fact that the top-condensate is driven solely by the bulk QCD gauge coupling which cannot be adjusted arbitrarily in this scenario. It is therefore completely different from the original version of the top-mode standard model, where the four-fermion coupling is introduced as an adjustable free parameter.

This fact is in sharp contrast to the renormalization group analysis of ACDH, where the cutoff $\Lambda$ is treated as an adjustable parameter of the model. Unfortunately, it is very difficult to perform such a quantitative calculation with sufficient reliability, however. We therefore do not discuss this problem hereafter in this paper.

It should also be noted that the cutoff $\Lambda$ needs to be fine-tuned to its critical value in order to obtain hierarchy between the cutoff $\Lambda$ and the fermion mass $B\left(M_{0}^{2}\right)$. The precise prediction of the cutoff and the order of fine-tuning depend on the detail of the model parameter, however. Actually, the scaling relation for $N_{f}=5$ (Fig, 13) indicates that the critical cutoff is much larger, $\Lambda^{2} \gtrsim 90\left|\left(\Lambda \frac{(D)}{\mathrm{MS}}\right)^{2}\right|$, in $N_{f}=5$ model.

\section{THE GAP EQUATION WITH USE OF THE EFFECTIVE COUPLING}

We have so far investigated the dynamical chiral symmetry breaking and the phase structure in the vector-like gauge theories with extra dimensions. Especially, we found that the simplest ACDH version of the top-mode standard model $\left(D=4+2, N_{f}=2\right)$ is in its chiral symmetric phase, indicating that the simplest ACDH scenario does not work properly as a model to explain the mass of the weak gauge bosons.

Our results, however, rely on our bold assumption, i.e., the non-perturbative existence of the UVFP. If the gauge coupling becomes stronger than our estimate of the UV-FP, there is a chance to obtain dynamical electroweak symmetry breaking even within the simplest ACDH model. Moreover, there is no justification to identify the renormalization scale $\mu^{2}$ of the $\overline{\mathrm{MS}}$ scheme with the gauge boson momentum $z \equiv-q^{2}$ beyond the leading order in the improved ladder approximation.

It is therefore worth analyzing the gap equation with use of different definition of the gauge coupling. Hereafter, we will investigate numerically the gap equation with use of the effective gauge coupling defined in Ref. [5]. The effective coupling is closely related to the gauge boson propagator and its momentum.

The effective gauge coupling $g_{\text {eff }}$ in the truncated KK effective theory on the 3-brane is given by

$$
\begin{aligned}
& \frac{-i}{g_{\mathrm{eff}}^{2}\left(-q^{2}\right)} D_{\mu \nu}^{-1}(q) \equiv \\
& \quad \frac{-i}{g_{0}^{2}} D_{(0) \mu \nu}^{-1}(q)-\left(q^{2} g_{\mu \nu}-q_{\mu} q_{\nu}\right) \Pi\left(q^{2}\right),
\end{aligned}
$$

with $g_{0}$ being bare gauge coupling of the truncated KK effective theory. $D_{\mu \nu}$ and $D_{(0) \mu \nu}$ are normalized

6 It is also possible to evaluate $v$ by adopting the Bardeen-Hill-Lindner (BHL) type compositeness condition 8 in the renormalization group analysis, however without freedom to adjust $\Lambda$. The decay constant $v$ is given by $v=\sqrt{2} m_{t} / y_{t}$ with $y_{t}$ being the Yukawa coupling satisfying the BHL condition. 
as

$$
\begin{aligned}
D_{\mu \nu}(q) & =\frac{-i}{q^{2}}\left(g_{\mu \nu}-\left(1-\xi\left(q^{2}\right)\right) \frac{q_{\mu} q_{\nu}}{q^{2}}\right), \\
D_{(0) \mu \nu}(q) & =\frac{-i}{q^{2}}\left(g_{\mu \nu}-\left(1-\xi_{0}\left(q^{2}\right)\right) \frac{q_{\mu} q_{\nu}}{q^{2}}\right) .
\end{aligned}
$$

Precise definitions of other notations are given in Ref. 胢 and the vacuum polarization function $\left(q^{2} g_{\mu \nu}-q_{\mu} q_{\nu}\right) \Pi\left(q^{2}\right)$ is evaluated using the background gauge fixing method so as to keep manifest gauge invariance. The vacuum polarization function $\Pi$ is the sum of loop contributions of each KKmodes. It includes not only logarithmically divergent contributions but also finite loop corrections.

Summing up the KK-mode contributions upto $m_{K K} \leq \Lambda_{g}$, we obtain a relation between effective and $\overline{\mathrm{MS}}$ couplings,

$$
\frac{1}{\hat{g}_{\mathrm{eff}}^{2}(z)}=\frac{\mu^{2}}{z}\left(\frac{1}{\hat{g}_{\overline{\mathrm{MS}}}^{2}(\mu)}-\frac{1}{g_{*}^{2}}\right)+\frac{1}{(4 \pi)^{3}}\left(K_{g}\left(-z, \Lambda_{g}^{2}\right)+K_{b}\left(-z, \Lambda_{g}^{2}\right)+K_{f}\left(-z, \Lambda_{g}^{2}\right)\right),
$$

at one-loop level in $D=4+2$ dimensions. Definitions of $K_{g}\left(q^{2}, \Lambda_{g}^{2}\right), K_{b}\left(q^{2}, \Lambda_{g}^{2}\right)$ and $K_{f}\left(q^{2}, \Lambda_{g}^{2}\right)$ are given in Appendix $\mathrm{D}$. We also defined dimensionless bulk gauge couplings $\hat{g}_{\text {eff }}^{2}(z)$ and $\hat{g}_{\overline{\mathrm{MS}}}(\mu)$ in a similar manner to Eq.(19),

$$
\hat{g}_{\mathrm{eff}}^{2}(z)=\frac{(2 \pi R \sqrt{z})^{2}}{n} g_{\mathrm{eff}}^{2}(z), \quad \hat{g}_{\overline{\mathrm{MS}}}^{2}(\mu)=\frac{(2 \pi R \mu)^{2}}{n} g_{\overline{\mathrm{MS}}}^{2}(\mu) .
$$

Plugging the solution of the $\overline{\mathrm{MS}}$ renormalization group equation Eq.(26) into Eq.(22), we can confirm the renormalization scale independence of the effective coupling,

$$
\frac{1}{\hat{g}_{\mathrm{eff}}^{2}(z)}=-\frac{1}{g_{*}^{2}} \frac{\left(\Lambda_{\left.\frac{(D)}{\mathrm{MS}}\right)^{2}}^{z}\right.}{z}+\frac{1}{(4 \pi)^{3}}\left(K_{g}\left(-z, \Lambda_{g}^{2}\right)+K_{b}\left(-z, \Lambda_{g}^{2}\right)+K_{f}\left(-z, \Lambda_{g}^{2}\right)\right) .
$$

The $\overline{\mathrm{MS}}$ gauge coupling in the ACDH scenario is in the weakly interacting region $\left(\Lambda_{\overline{\mathrm{MS}}}^{(D)}\right)^{2}<0$.

It should be emphasized that the effective coupling Eq.(72) depends on the choice of cutoff $\Lambda_{g}$, no matter how large it is. This behavior implies the violation of the decoupling theorem. The low energy $\left(\ll \Lambda_{g}\right)$ predictions are sensitive to the physics at the cutoff scale. There is no UV-FP in the usual sense due to the violation of the decoupling theorem, although there still exists an upper bound of $\hat{g}_{\text {eff }}$ if $\left(\Lambda \frac{(D)}{\mathrm{MS}}\right)^{2} \leq 0$,

$$
\hat{g}_{\mathrm{eff}}^{2}(z) \leq\left.\hat{g}_{\mathrm{eff}}^{2}\left(z=\Lambda_{g}^{2}\right)\right|_{\left(\Lambda \frac{(D)}{\mathrm{MS}}\right)^{2}=0}=\frac{(4 \pi)^{3}}{K}
$$

with

$$
K \equiv K_{g}\left(-\Lambda_{g}^{2}, \Lambda_{g}^{2}\right)+K_{b}\left(-\Lambda_{g}^{2}, \Lambda_{g}^{2}\right)+K_{f}\left(-\Lambda_{g}^{2}, \Lambda_{g}^{2}\right),
$$

where we identified $\Lambda_{g}$ with the cutoff for the gauge boson propagator. The factor $K$ is evaluated in $S U(N)$ gauge theory with $N_{f}$ flavors,

$$
K \equiv N\left(-\frac{88}{45}+\frac{10 \sqrt{5}}{3} \operatorname{arctanh} \frac{1}{\sqrt{5}}\right)-\frac{32}{45} N_{f} \simeq 1.63 N-0.71 N_{f} .
$$

We note that the upper bound of $\hat{g}_{\text {eff }}^{2}$ is roughly twice larger than the corresponding $\overline{\mathrm{MS}}$ UV-FP. 


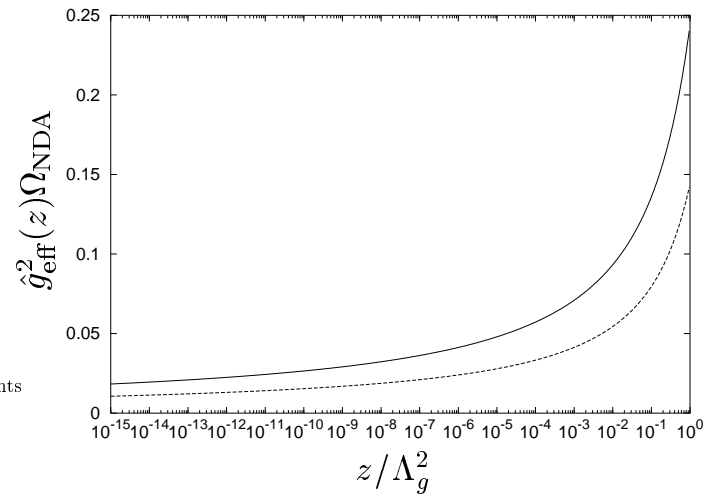

FIG. 14: The typical flow of the effective coupling $\hat{g}_{\text {eff }}^{2}$ defined by Eq. (74) with $\left(\Lambda \frac{(D)}{\mathrm{MS}}\right)^{2}=0$. The dashed and solid lines represents the graphs for $N_{f}=2,4$, respectively. In both graphs, we took $N=3$, and $\left(\Lambda_{g} R\right)^{2}=4 \times 10^{10}$.

Fig. 14 shows typical behavior of the effective coupling $\hat{g}_{\text {eff }}^{2}$ with $\left(\Lambda \frac{(D)}{\mathrm{MS}}\right)^{2}=0$. Substituting the effective coupling shown in Fig. 14 into the formula of the non-local gauge fixing Eq.(16), we obtain the corresponding behaviors of the non-local gauge fixing function $\xi(z)$. (See Fig. 15.)

In order to solve the gap equation, we first recall Eq.(12), the relation between the gauge boson momentum $z$ and the fermion momenta $x, y$ in the gap equation. The gauge boson momentum $z$ reaches its maximum $4 \Lambda^{2}$ when $x=y=\Lambda^{2}$ and $\cos \theta=-1$ in Eq.(12). The cutoff of the gauge boson momentum $\Lambda_{g}^{2}$ thus needs to satisfy

$$
\Lambda_{g}^{2} \geq 4 \Lambda^{2}
$$

Hereafter we simply assume $\Lambda_{g}^{2}=4 \Lambda^{2}$ unless noted otherwise.

It is difficult fully to take into account the effect of the compactification scale $R^{-1}$. We introduce an infrared cutoff $M_{0}^{2} \sim R^{-2}$ in the gap equation and neglect $R^{-1}$ sensitive infrared behaviors in the following analysis instead. We find the dynamical chiral symmetry breaking is insensitive to $M_{0}$ for sufficiently large $\Lambda$ anyway.

The minimal ACDH scenario corresponds to $S U(3)$ gauge theory in the $D=4+2$ dimensional spacetime with $N_{f}=2$ and $\left(\Lambda \frac{(D)}{\mathrm{MS}}\right)^{2}<0$. The effective gauge coupling of the ACDH scenario $\left(\Lambda \frac{(D)}{\mathrm{MS}}\right)^{2}<0$ is always weaker than the case with $\left(\Lambda \frac{(D)}{\mathrm{MS}}\right)^{2}=0$. It is therefore sufficient to investigate the case $\left(\Lambda \frac{(D)}{\mathrm{MS}}\right)^{2}=0$ for the determination of the condition of the bulk chiral symmetry breaking. The aim of our numerical analysis is then to find the critical $N_{f}$, above which the dynamical chiral symmetry breaking takes place in the bulk with $\left(\Lambda_{\frac{(D)}{\mathrm{MS}}}\right)^{2}=0$. We take the chiral limit $m_{0}=0$ in the following analyses.

It is now easy to perform numerical analysis of the gap equation by using the recursion method [5]. Hereafter, we formally admit $N_{f}$ to take non-integer (real) value and evaluate the scaling behavior of $B\left(M_{0}^{2}\right)$ as a function of $N_{f}$. For $S U(3)$ gauge theory in $D=4+2$ dimensions, we obtain the scaling behavior shown in Fig.16. The dynamical chiral symmetry breaking takes place for

$$
N_{f}>N_{f}^{\text {crit }}=4.23 \quad \text { with }\left(\Lambda_{g} / \Lambda\right)^{2}=4
$$

in this model.

For $\Lambda_{g}^{2}>4 \Lambda^{2}$, critical $N_{f}$ tends to be lager than Eq. (79). For instance, we obtain

$$
N_{f}^{\text {crit }}=4.62 \quad \text { with }\left(\Lambda_{g} / \Lambda\right)^{2}=10 \text {. }
$$




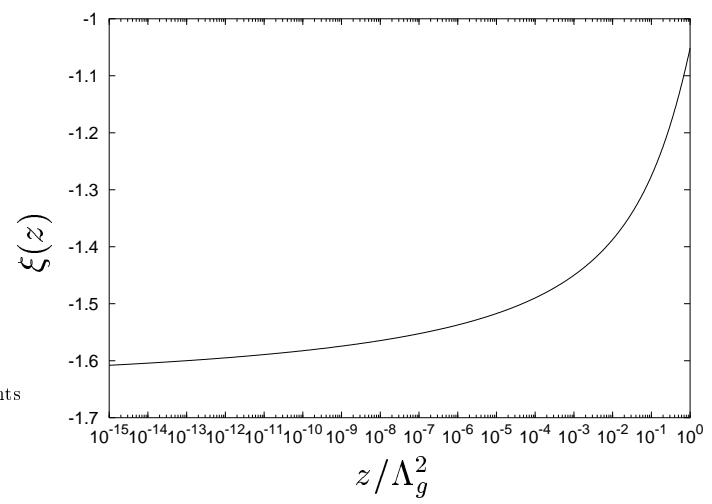

FIG. 15: The typical behavior of the NLG $\xi(z)$ for the effective coupling defined by Eq. (74) with $\left(\Lambda_{\overline{\mathrm{MS}}}^{(D)}\right)^{2}=0$. We took $N=3,\left(\Lambda_{g} R\right)^{2}=4 \times 10^{10}$, and $N_{f}=4$. The graph for $N_{f}=2$ almost overlaps with the above one. The NLG approaches $\xi=-5 / 3$ in the limit of $z \rightarrow 0$.

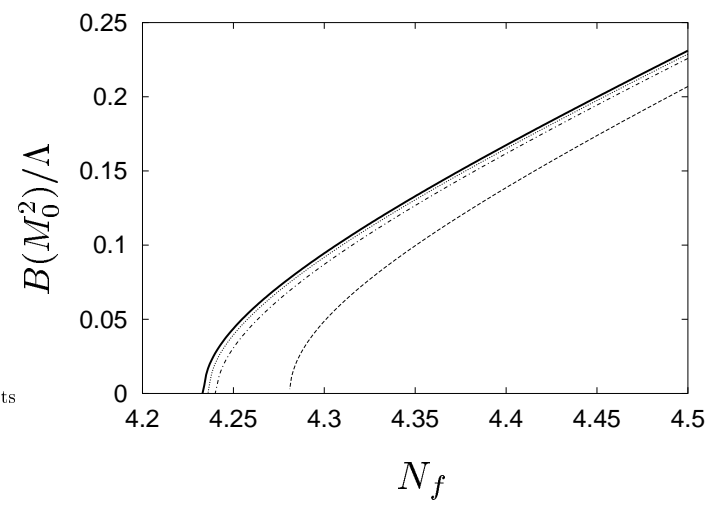

FIG. 16: The scaling behavior for the dynamical mass with the effective coupling $\hat{g}_{\text {eff }}^{2}$ The lines from right to left are graphs for $\left(\Lambda / M_{0}\right)^{2}=10^{2}, 10^{3}, 10^{5}, 10^{10}$ with $N=3$ and $\left(\Lambda_{g} / \Lambda\right)^{2}=4$, respectively.

The physics behind this result is obviously understood if we note Eq.(75). The effective coupling does not reach its maximum value in the gap equation for $\Lambda_{g}^{2}>4 \Lambda^{2} \geq z$. We thus conclude that Eq.(79) is very conservative estimate and that the simplest version of the ACDH model with $N_{f}=2$ does not work even with the effective coupling.

Noting the upper bound of $\hat{g}_{\text {eff }}^{2}$ is approximately twice larger than the UV-FP of $\hat{g}_{\overline{\mathrm{MS}}}^{2}$, it is somewhat surprising to find that $N_{f}^{\text {crit }}$ with the effective coupling Eq. $(79)$ is rather close to $N_{f}^{\text {crit }}$ with the $\overline{\mathrm{MS}}$ coupling Eq.(56). It should be emphasized that Fig.14 shows the effective coupling $\hat{g}_{\text {eff }}^{2}$ is close to its maximum value only when the gauge boson momentum $z$ is sufficiently close to $\Lambda_{g}^{2}$, however. Unlike the corresponding UV-FP of the $\overline{\mathrm{MS}}$ coupling, the effective coupling is well below its maximum value in the wide region of momentum space.

Actually, similar situation was also found in the analysis of four-dimensional QED with including vacuum polarization effects [32]. In the case of $N_{f}=1 \mathrm{QED}_{4}$, the dynamical chiral symmetry breaking takes place only when the coupling at the cutoff exceeds the critical value

$$
\alpha_{\Lambda}>1.95, \quad\left(N_{f}=1\right)
$$

which is about twice larger than the quenched one $\left(\alpha_{c}=\pi / 3\right)$. 
We finally make a brief comment on the scaling relation. Unlike the essential-singularity type scaling Eq.(54) found in the analysis with the $\overline{\mathrm{MS}}$ coupling, the scaling behavior of Fig. 16 seems like the mean-field type scaling. In order to confirm the mean-field type scaling, we perform a fit of the scaling behavior assuming power-low scaling,

$$
B\left(M_{0}^{2}\right) \propto \Lambda\left(N_{f}-N_{f}^{\text {crit }}\right)^{\gamma},
$$

in Fig.17. We find the best fit value $\gamma$ is given by

$$
\gamma=0.51
$$

for the data set with

$$
\frac{N_{f}-N_{f}^{\text {crit }}}{N_{f}^{\text {crit }}} \leq 0.01
$$

This result is consistent with the mean-field type scaling $\gamma=1 / 2$. In contrast to the case with the essential-singularity type scaling, the cutoff $\Lambda$ needs to be small enough to keep the dynamical mass small even when $N_{f}$ is sufficiently close to $N_{f}^{\text {crit }}$ with the mean-field type scaling.

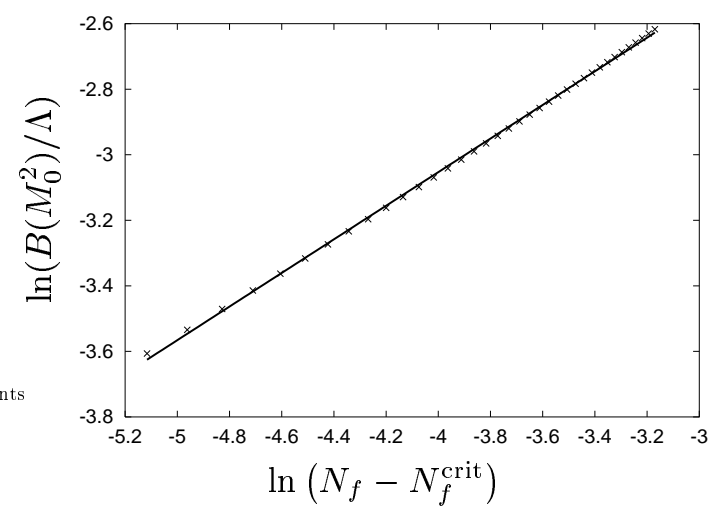

FIG. 17: The log-log plot of the scaling relation Fig. 16. We used $\left(\Lambda / M_{0}\right)^{2}=10^{10}$ and $\left(\Lambda_{g} / \Lambda\right)^{2}=4$. The bold line represents the line obtained through the least-squares method for the data set of $\left(N_{f}-N_{f}^{\text {crit }}\right) / N_{f}^{\text {crit }}<0.01$ with $N_{f}^{\text {crit }}=4.23$. We also plot numerical data with crossed points.

\section{SUMMARY AND DISCUSSIONS}

In this paper, we have systematically studied the bulk dynamical chiral symmetry breaking in vector-like gauge theories with extra dimensions and revealed a new phase structure of such theories. Extending our previous study based on the gap equation (the SD equation within the improved ladder approximation), we adopted in the present study the non-local gauge fixing method in order to keep the $\not p$ part of the fermion propagator to be trivial, i.e., $A\left(-p^{2}\right) \equiv 1$, which is thus consistent with the Ward-Takahashi identity and the bare vertex approximation in the ladder SD equation.

The one-loop analysis of the $\overline{\mathrm{MS}}$ beta function suggests the existence of a ultra-violet fixed-point (UV-FP) $g_{*}$ in the truncated KK effective theory of a non-Abelian gauge theory with compactified extra dimensions. The existence of such a UV-FP may open interesting possibilities in the model building of the high-energy particle theory. The top mode standard model (TMSM) scenarios in extra dimensions, for example, are affected significantly by the existence of such a UV-FP. It is therefore interesting to investigate consequences of a UV-FP in the bulk field theories. We therefore 
first analyzed the gap equation with the gauge coupling both on and off the UV-FP, assuming the qualitative structure of the UV-FP is unchanged beyond the one-loop approximation. We found that the critical UV-FP gauge coupling is $D / 4$ times larger than our previous calculation in the Landau gauge. The result was then converted to critical number of flavors $N_{f}^{\text {crit }}$. For $N_{f}>N_{f}^{\text {crit }}$ the dynamical chiral symmetry breaking takes place not only in the "strong-coupling phase" $\hat{g}>g_{*}$, but also in the "weak-coupling phase" $\hat{g}<g_{*}$ when the cutoff is large enough. For $N_{f}<N_{f}^{\text {crit }}$, however, the chiral symmetry remains unbroken in the "weak-coupling phase" $\hat{g}<g_{*}$ no matter how large the cutoff is. We found $N_{f}^{\text {crit }} \simeq 4.2,1.8$ in $D=6,8$ dimensions for the $S U(3)$ gauge theory (bulk QCD).

In a scenario with the extra dimensions (ACDH scenario) of the TMSM, the gauge coupling is obviously weak and the $N_{f}$ needs to be larger than the critical one in order to trigger the dynamical electroweak symmetry breaking. The simplest ACDH scenario with $N_{f}=2$ thus does not work in $D=6$, while there is a chance to construct a viable model in $D=8$ dimensions. Moreover, the UV cutoff needs to be finite in order to obtain finite top quark mass. Actually, once we fix the top mass, it is possible to predict the UV cutoff in the ACDH scenario in the analysis of the gap equation, in contrast to the original treatment of ACDH. The phenomenological analysis done by ACDH therefore needs to be modified by taking account of this fact.

On the other hand, we found a novel situation for $N_{f}<N_{f}^{\text {crit }}$, where we can formally define a continuum limit (infinite cutoff). The low energy physics is controlled by the properties of the UVFP and it becomes insensitive to the physics around the UV cutoff. The anomalous dimension of the fermion mass $\gamma_{m}$ is shown to be large. This phase may be useful for model building of "bulk technicolor", where the large anomalous dimension can be used as a suppression mechanism of the excess of FCNC.

It should be emphasized, however, that non-perturbative existence of UV-FP is no more than an assumption at present in a wide class of models. Actually, the one-loop effective gauge coupling of the truncated KK effective theory with $D=4+2$ dimensions is shown to have an explicit cutoff dependence, which implies absence of the UV-FP in the usual sense. We therefore performed an analysis of the gap equation by using the effective coupling for $D=4+2$ dimensions. We found that there also exists a $N_{f}^{\text {crit }}$, notwithstanding absence of UV-FP in the usual sense. Although the effective coupling at the UV cutoff is much larger than that of $\overline{\mathrm{MS}}$, we found that the $N_{f}^{\text {crit }}$ in this scheme actually is very close to the $\overline{\mathrm{MS}}$ one. The simplest version of ACDH scenario with $N_{f}=2$ is therefore quite unlikely to work in the $D=4+2$ dimensions.

Many issues remain unsolved and need further study, however. For a example, the existence of a non-trivial UV-FP is yet to be proved. It should be investigated more definitely in future whether the non-trivial UV-FP really exists or not.

The uncertainties coming from compactification sensitive infrared (IR) region are also important. In the present paper, this effect was only roughly estimated by introducing the IR cutoff $M_{0}^{2} \sim R^{-2}$ in the gap equation. It turned out that the effects tend to increase the critical coupling or $N_{f}^{\text {crit }}$. Hence there is a possibility that the simplest version of the ACDH scenario may not work even for $D=4+4$. We need to invent more sophisticated way to take into account the compactification effect particularly for the case $B_{0} \ll R^{-1}$.

Finally, the results presented in this paper provide basic tools for the particle model building with the dynamical chiral symmetry breaking in extra dimensions. We need to construct concrete and viable models such as top-condensate or technicolor in the bulk by using these tools. More quantitative studies on these models will be dealt in a separated publication.

\section{Acknowledgments}

This work is supported by Grant-in-Aid for Scientific Research (B) \#11695030 (K.Y., V.G.), the JSPS Research Fellowships for Young Scientists \#01170 (M.H.), and partially by the grant SCOPES project 7 IP 062607 of the Swiss NSF (V. G.). V.G. wishes to acknowledge JSPS for financial support. 


\section{APPENDIX A: THE EFFECTIVE POTENTIAL FOR LOCAL COMPOSITE FIELD}

We expect appearance of various composite $\bar{\psi} \psi$ bound states in the gauge theories with extra dimensions. The $\sigma$, chiral partner of the Nambu-Goldstone boson, is particularly important among them, because it corresponds to the Higgs boson in the models of the dynamical electroweak symmetry breaking. However, the CJT potential discussed in section III is a functional of the mass function $B(x)$, and is not directly related to these bound states. In addition, it is shown that the CJT is not bounded from below. It is therefore not perfectly appropriate to study the stability of the vacuum by using the CJT potential.

In this Appendix, we thus discuss yet another effective potential $V(\sigma)$, which is a function of the local composite field $\sigma \sim \bar{\psi} \psi$ and is connected to the dynamical properties of the $\sigma$ boson more closely.

We consider the effective action $\Gamma[\sigma]$,

$$
\Gamma[\sigma] \equiv W[J]-\int d^{D} x J \sigma
$$

with

$$
W[J] \equiv \frac{1}{i} \ln \int[d \psi d \bar{\psi}][\text { gauge }] \exp \left(i \int d^{D} x(\mathcal{L}+J \bar{\psi} \psi)\right), \quad \sigma \equiv \frac{\partial W[J]}{\partial J} .
$$

The corresponding effective potential can be obtained by taking the coordinate independent part of this effective action.

In the following, we briefly outline the derivation of the effective potential $V(\sigma)$ based on the method of Ref. [33] (see also Ref. [16]).

For a constant source term $J$, the partition function $W[J]$ is obtained as

$$
W[J]=\int d J \frac{\partial W[J]}{\partial J} \int d^{D} x .
$$

Noting

$$
\frac{\partial W[J]}{\partial J}=\langle\bar{\psi} \psi\rangle_{J}=\sigma,
$$

we find the effective potential is given by

$$
V(\sigma)=J \sigma-\int^{J} d J \sigma\left(=\int^{\sigma} d \sigma J\right)
$$

where $J$ should be regarded as a function of $\sigma$.

The effect of the constant source $J$ can be obtained by replacing the bare mass $m_{0}$ in Eqs.(31), (35), (45) and (48),

$$
m_{0} \rightarrow m_{0}-J
$$

We thus find

$$
J=m_{0}-\frac{1}{2}(1+\tilde{\nu}) \tilde{c}_{0} B_{0}\left(\frac{\Lambda^{2}}{B_{0}^{2}}\right)^{-\omega(1-\tilde{\nu})}
$$

for $\kappa_{D}<\kappa_{D}^{\text {crit }}$ and

$$
J=m_{0}-\sqrt{1+\nu^{2}}\left|c_{0}\right| B_{0}\left(\frac{\Lambda^{2}}{B_{0}^{2}}\right)^{-\omega} \sin \left[\theta+\omega \nu \ln \frac{\Lambda^{2}}{B_{0}^{2}}+\tan ^{-1} \nu\right],
$$


for $\kappa_{D}>\kappa_{D}^{\text {crit }}$. The relation between the mass function $B(x)$ and $\sigma$ is given by

$$
\sigma=\langle\bar{\psi} \psi\rangle_{J}=-\eta N N_{f} \Omega_{\mathrm{NDA}} \int_{0}^{\Lambda^{2}} d x x^{D / 2-1} \frac{B(x)}{x+B^{2}}
$$

which leads to

$$
\sigma=\langle\bar{\psi} \psi\rangle_{J}=\left.\eta N N_{f} \Omega_{\mathrm{NDA}} \frac{\kappa_{D}^{\mathrm{crit}}}{\omega^{2} \kappa_{D}} \Lambda^{D} \frac{d B(x)}{d x}\right|_{x=\Lambda^{2}},
$$

where we have used the gap equation Eq.(31). The chiral condensate $\sigma$ can be expressed in terms of $B_{0}$,

$$
\sigma\left(B_{0}\right)=-\tilde{c}_{0} \eta N N_{f} \Omega_{\mathrm{NDA}} \frac{(1-\tilde{\nu}) \kappa_{D}^{\mathrm{crit}}}{\omega \kappa_{D}} \Lambda^{D-1}\left(\frac{\Lambda}{B_{0}}\right)^{-2 \omega(1-\tilde{\nu})-1}
$$

for $\kappa_{D}<\kappa_{D}^{\text {crit }}$ and

$$
\sigma\left(B_{0}\right)=-\frac{2\left|c_{0}\right| \eta N N_{f} \Omega_{\mathrm{NDA}}}{\omega \sqrt{1+\nu^{2}}} \Lambda^{2 \omega} B_{0}^{2 \omega+1} \sin \left[\theta+\omega \nu \ln \frac{\Lambda^{2}}{B_{0}^{2}}-\tan ^{-1} \nu\right]
$$

for $\kappa_{D}>\kappa_{D}^{\text {crit }}$.

We are now ready to evaluate the effective potential Eq. A5). The $J$ (or $\sigma$ ) integral in Eq. A5) can be performed by using

$$
d J=d B_{0} \frac{d J}{d B_{0}}, \quad\left(\text { or } \quad d \sigma=d B_{0} \frac{d \sigma}{d B_{0}}\right) .
$$

Combining Eqs. (A5), (A7), (A10) and (A11), we finally obtain the effective potential in the subcritical region $\kappa_{D}<\kappa_{D}^{\text {crit }}$

$$
V(\sigma)=\frac{1}{D-2} \eta N N_{f} \Omega_{\mathrm{NDA}} \tilde{c}_{0}^{2} \Lambda^{D}\left(\frac{B_{0}^{2}}{\Lambda^{2}}\right)^{1+2 \omega(1-\tilde{\nu})},
$$

in the chiral limit $m_{0}=0$. The effective potential in the super-critical region can be obtained in a similar manner,

$$
V(\sigma)=\frac{2}{D-2} \eta N N_{f} \Omega_{\mathrm{NDA}}\left|c_{0}\right|^{2} B_{0}^{D}\left[-\cos \left(2 \theta+2 \omega \nu \ln \frac{\Lambda^{2}}{B_{0}^{2}}\right)+A\right],
$$

with

$$
A \equiv \frac{\kappa_{D}^{\mathrm{crit}}}{\kappa_{D}}\left(1-\frac{1+6 \omega}{1+2 \omega} \nu^{2}\right)
$$

We regard here $B_{0}$ as an function of $\sigma$ defined implicitly in Eq. A10).

It is now straightforward to find the stationary points of the effective potential. The stationary condition $d V / d \sigma=0$ of Eq. A14 has only the trivial solution $B_{0}=0$ for $\kappa_{D}<\kappa_{D}^{\text {crit }}$, while we find non-trivial solutions $B_{0}=\widehat{B_{0}^{(n)}} \neq 0(n=1,2, \cdots)$ for $\kappa_{D}>\kappa_{D}^{\text {crit }}$ in the stationary condition of Eq. (A15). Here, $B_{0}^{(n)}$ is given by

$$
B_{0}^{(n)} \equiv \Lambda \exp \left[\frac{-n \pi+\theta+\tan ^{-1} \nu}{2 \omega \nu}\right],
$$

which coincides with the solution of Eq.(48) with $m_{0}=0$. 
Hereafter, we concentrate on the supercritical region $\kappa_{D}>\kappa_{D}^{\text {crit }}$. The stability of the vacua $(n=$ $1,2, \cdots)$ can be investigated by taking the second derivative of the potential,

$$
\frac{d^{2} V}{d \sigma^{2}}=\frac{d J}{d \sigma}=\frac{d J}{d B_{0}} \cdot\left(\frac{d \sigma}{d B_{0}}\right)^{-1}
$$

It is easy to show that the curvature of the potential at stationary point is positive

$$
\left.\frac{d^{2} V}{d \sigma^{2}}\right|_{\sigma=\sigma\left(B_{0}^{(n)}\right)}=\frac{\omega^{2}\left(1+\nu^{2}\right)}{2\left(1+3 \omega-\omega \nu^{2}\right) \eta N N_{f} \Omega_{\mathrm{NDA}}} \Lambda^{-(D-2)}>0
$$

for $\nu^{2}<3+\omega^{-1}$ irrespective of $n$. We thus find that every stationary point is a local minimum of the potential $V(\sigma)$.

We need to compare vacuum energies in order to find the absolute minimum of the potential, i.e., the true vacuum then. The value of the potential at each $n$ is obtained as

$$
V\left(B_{0}^{(n)}\right)=-\frac{4}{D}\left(1-\frac{\kappa_{D}^{\text {crit }}}{\kappa_{D}}\right)\left|c_{0}\right|^{2} \eta N N_{f} \Omega_{\mathrm{NDA}}\left(B_{0}^{(n)}\right)^{D}<0
$$

which is actually consistent with the result of the CJT potential Eq. (51) for small $B_{0}^{(n)}$. The $n=1$ solution, i.e., the largest fermion mass, gives the global minimum of $V(\sigma)$. We thus conclude that the $n=1$ solution corresponds to the most stable vacuum.

We should comment here on the properties of the false vacua $n \geq 2$. Although we found that the mass square of $\sigma$ is positive even in these false vacua, it does not necessarily imply the meta-stability of these vacua. Actually, in the analysis of the Bethe-Salpeter equations in the strong coupling $\mathrm{QED}_{4}$ (QED in four dimensions), it is known that these false vacua have tachyonic mode(s) in the pseudoscalar channel in addition to the massless Nambu-Goldstone mode [28, 35]. The false vacua $n \geq 2$ are therefore saddle points of the effective potential with negative curvature in the direction of the pseudoscalar channel when pseudoscalar degrees of freedom are included in the potential.

As we described before, we found that the every stationary point of the potential Eq.(A15) is local minimum in the $\sigma$-direction. There is no stationary point corresponding to local maximum. This fact perhaps may sound rather peculiar. Actually, it is tied with the interesting and bizarre property of the effective potential Eq. A15). The effective potential Eq.A15) is a multi-branched and multi-valued function of $\sigma$. We next try to grasp the shape of the effective potential more closely.

We first consider the derivatives of $\sigma$ (Eq.(A12)) and $J$ (Eq.(A8)) with respect to $B_{0}$ :

$$
\frac{d \sigma}{d B_{0}} \propto \Lambda^{2 \omega} B_{0}^{2 \omega} \sin \left(\theta+\omega \nu \ln \frac{\Lambda^{2}}{B_{0}^{2}}-\tan ^{-1}\left[\frac{2(D-1) \nu}{D-(D-2) \nu^{2}}\right]\right)
$$

and

$$
\frac{d J}{d B_{0}} \propto \Lambda^{-2 \omega} B_{0}^{2 \omega} \sin \left(\theta+\omega \nu \ln \frac{\Lambda^{2}}{B_{0}^{2}}+\tan ^{-1}\left[\frac{2 \nu}{D+(D-2) \nu^{2}}\right]\right) .
$$

We note that $d \sigma / d B_{0}$ vanishes for

$$
\theta+\omega \nu \ln \frac{\Lambda^{2}}{B_{0}^{2}}-\tan ^{-1}\left(\frac{2(D-1) \nu}{D-(D-2) \nu^{2}}\right)=n \pi, \quad n=1,2, \cdots
$$

while $d J / d B_{0}$ remains finite for $B_{0}$ with Eq.(A22). The second derivative of the potential Eq. A17) thus diverges at Eq. A22). We note here, however, the first derivative of the potential $d V / d \sigma(=$ $\left.d V / d B_{0} \cdot d B_{0} / d \sigma\right)$ remains finite at Eq. A22) despite $d V / d B_{0}=0$, since there exists in $d V / d B_{0}$ the same sine-function as in $d \sigma / d B_{0}$. There should take place something very bizarre at the points Eq. A22). 
Plotting the shape of the effective potential, we find that it has structure quite similar to the potential Fig.1 of Ref. [34], in which the diquark condensate is studied in high density QCD by using the local composite effective potential. The potential $V(\sigma)$ is a multi-branched and multi-valued function of $\sigma$ : The points Eq. A22 are cusps and correspond to branching points. The stationary point $\sigma\left(B_{0}^{(n)}\right)$ corresponds to the local minimum in each branch of the effective potential. We also find easily from Eqs. (A22) and (A12) that the branching point converges to $\sigma=0$ in the $n \rightarrow \infty$ limit. This means that the branch of the potential also shrinks into $\sigma=0$, exhibiting a fractal structure around $\sigma=0$.

It is known that the long-range nature of interactions in scale invariant theories also leads to other peculiar properties such as the existence of the infinite number of resonances and the non-analyticity of the potential at the point $\sigma\left(B_{0}=0\right)$ causing its fractal structure around $\sigma\left(B_{0}=0\right)$ [34]. It should be noted, however, that the gauge theories with extra dimensions are not scale invariant below the compactification scale which should serve as an infrared cutoff in the SD equation for the fermion mass function. Introducing such a cutoff explicitly, one can show that the SD equation has only finite number of solutions (see Refs. [35, 36] and Appendix $\mathrm{C}$ of the present paper) and the solutions with small dynamical masses $B_{0}^{(n \gg 1)}$ disappear. Accordingly, the potential $V(\sigma)$ will have only finite number of branches. We thus expect that bizarre behavior near $\sigma=0$ does not actually occur in models treated in this paper.

Finally, we discuss the properties of the effective potential for sufficiently large cutoff $\Lambda \gg B_{0}^{(1)}$. For such a purpose, we take an infinite cutoff limit $\Lambda \rightarrow \infty$ with $B_{0}^{(1)}$ being fixed by formally adjusting $\nu \equiv \sqrt{\kappa_{D} / \kappa_{D}^{\text {crit }}-1}$. The anomalous dimension of the fermion mass is found to be $\gamma_{m}=2 \omega$ in Ref. [5] in such a formal limit. We thus define a "renormalized" operator,

$$
(\bar{\psi} \psi)_{R} \equiv Z_{m}(\bar{\psi} \psi), \quad Z_{m} \propto\left(\frac{\mu}{\Lambda}\right)^{2 \omega}
$$

and

$$
\sigma_{R} \equiv\left\langle(\bar{\psi} \psi)_{R}\right\rangle_{J}
$$

Taking the formal $\Lambda \rightarrow \infty$ limit of Eq. A12 as described before, it is easy to obtain

$$
\sigma_{R} \propto \mu^{2 \omega} B_{0}^{D / 2}\left(\frac{1}{\omega}+\ln \frac{B_{0}}{B_{0}^{(1)}}\right) .
$$

As expected from the argument of the anomalous dimension, $\sigma_{R}$ remains finite even in this formal $\Lambda \rightarrow \infty$ limit.

Eq. (A25) can be used to define $B_{0}$ as a function of $\sigma_{R}$. We are thus able to rewrite the effective potential Eq. A15 as a function of the renormalized field, $V_{R}\left(\sigma_{R}\right)$. In the formal $\Lambda \rightarrow \infty$ limit, we obtain

$$
V_{R}\left(\sigma_{R}\right)=\eta N N_{f} \Omega_{\mathrm{NDA}} \frac{[\Gamma(D / 2)]^{2}}{[\Gamma(1+\omega)]^{4}} B_{0}^{D}\left[\omega\left(\ln \frac{B_{0}}{B_{0}^{(1)}}\right)^{2}+\ln \frac{B_{0}}{B_{0}^{(1)}}-\frac{1}{D}\right],
$$

with $B_{0}=B_{0}\left(\sigma_{R}\right)$ being the function of $\sigma_{R}$ determined implicitly from Eq.A25.

It is somewhat surprising to find such a finite expression of the effective potential Eq. A26) in the $\Lambda \rightarrow \infty$ limit in the non-renormalizable higher-dimensional gauge theories. This property is actually related to the approximate scale invariance, i.e., the existence of the UV-FP.

\section{APPENDIX B: CONVERSION TO THE SCHRÖDINGER-LIKE EQUATION}

We discuss the dynamical mass generation in the bulk from a little bit different point of view. The $\mathrm{SD}$ eq. (31) for the mass function without the bare mass term $m_{0}$ can be rewritten in a form of the 
Schrödinger-like equation [37], in which the $\mathrm{D} \chi \mathrm{SB}$ takes place when a "bound state" exists. The subject whether the bulk fermion condenses or not is, thus, reduced to the "bound state problem" in the quantum mechanics.

Let us start with introducing the "wave function"

$$
\psi(u) \equiv \int \frac{d^{D} q_{E}}{(2 \pi)^{D}} \frac{e^{i q_{E} \cdot u} B\left(q_{E}^{2}\right)}{q_{E}^{2}+m^{2}} .
$$

The Fourier transform of the mass function $B$ is then given by

$$
\int \frac{d^{D} q_{E}}{(2 \pi)^{D}} e^{i q_{E} \cdot u} B\left(q_{E}^{2}\right)=\left(-\triangle_{u}+m^{2}\right) \psi(u)
$$

We next consider a linearized version of the ladder SD equation,

$$
B\left(p_{E}^{2}\right)=C_{F} \int \frac{d^{D} q_{E}}{(2 \pi)^{D}} \frac{B\left(q_{E}^{2}\right)}{q_{E}^{2}+m^{2}} \frac{(D-1+\xi) g_{*}^{2}}{\left(p_{E}-q_{E}\right)^{2(D / 2-1)}},
$$

where the coupling $g_{D}^{2}$ is replaced by the running one $g_{*}^{2} /\left(p_{E}-q_{E}\right)^{2(D / 2-2)}$ and we take $m=B(0)$. It is straightforward to show that the Fourier transform of Eq.(B3) is formally given by

$$
\int \frac{d^{D} q_{E}}{(2 \pi)^{D}} e^{i q_{E} \cdot u} B\left(q_{E}^{2}\right)=-V(u) \psi(u),
$$

where the "potential" $V(u)$ is defined by

$$
V(u) \equiv-\left(D-1+\xi_{D}\right) C_{F} g_{*}^{2} \int \frac{d^{D} p_{E}}{(2 \pi)^{D}} \frac{e^{i p_{E} \cdot u}}{\left[p_{E}^{2}\right]^{D / 2-1}}
$$

and the gauge fixing parameter $\xi$ is taken to the value of Eq. 29, ,

$$
\xi_{D} \equiv-\frac{(D-1)(D-4)}{D} .
$$

Here, the momentum shift invariance $\left(p_{E} \rightarrow p_{E}-q_{E}\right)$ is assumed ${ }^{7}$. Eqs. (B2) and (B4) then lead to the Schrödinger-like equation,

$$
H \psi(u)=E \psi(u)
$$

with

$$
H \equiv-\triangle_{u}+V(u), \quad E \equiv-m^{2} .
$$

Namely, non-trivial solutions of the SD equation correspond to bound states $(E<0)$ in the Schrödinger-like equation.

In order to solve the Schrödinger-like equation (B7), we rewrite it in the spherical coordinate. The "wave function" is decomposed as

$$
\psi(u) \equiv r^{-\frac{D-1}{2}} R(r) X\left(\phi_{i}\right), \quad r=|u|,
$$

\footnotetext{
7 It should be noted, however, the UV-cutoff $\Lambda$ in the SD equation violates the momentum shift invariance. The analysis of the Schrödinger-like equation can thus be regarded as an analysis of the SD equation with a different choice of the UV-cutoff procedure.
} 
where $R(r)$ and $X\left(\phi_{i}\right)$ denote the radial function and an analogue of the spherical surface harmonics in $D$-dimensions (the Gegenbauer function), respectively. For a "S-wave wave function" with $X\left(\phi_{i}\right) \equiv 1$, the $D$-dimensional Laplacian can be written as

$$
\begin{aligned}
& \triangle_{u} \psi_{\mathrm{S}}(u)= \\
& \frac{1}{r^{D-1}} \frac{\partial}{\partial r}\left[r^{D-1} \frac{\partial}{\partial r}\left(r^{-\frac{D-1}{2}} R(r)\right)\right] .
\end{aligned}
$$

It is straightforward to show that Eq. (B7) leads to

$$
\left[-\frac{\partial^{2}}{\partial r^{2}}+V_{\mathrm{eff}}(r)\right] R(r)=E R(r)
$$

with

$$
V_{\mathrm{eff}}(r) \equiv V(r)+\frac{(D-1)(D-3)}{4} \frac{1}{r^{2}} .
$$

We find that an additional "positive centrifugal potential" appears from the kinetic term in the case of $D>3$ even if we consider the S-wave solution.

The "potential" $V(r)$ in Eq. (B5) also has the same power of $r$ (an attractive inverse square "potential"):

$$
V(r)=-\frac{(D-2)^{2}}{4} \frac{\kappa_{D}}{\kappa_{D}^{\text {crit }}} \frac{1}{r^{2}} .
$$

The competition between the "repulsive centrifugal potential" and the "attractive inverse square potential" thus determines the dynamical symmetry breaking.

The bound state spectrum with an inverse square potential can be found in various textbooks of quantum mechanics [38]. The equation

$$
\frac{\partial^{2} R(r)}{\partial r^{2}}+\left[\epsilon+\frac{\alpha}{r^{2}}\right] R(r)=0,
$$

has an infinite number of bound state solutions only when $\alpha>1 / 4$. In the present case, the parameters $\epsilon$ and $\alpha$ are given by

$$
\epsilon=-m^{2}, \quad \alpha=\frac{(D-2)^{2}}{4}\left(\frac{\kappa_{D}}{\kappa_{D}^{\text {crit }}}-1\right)+\frac{1}{4} .
$$

The bound states of the Schrödinger-like equation exist if and only if $\kappa_{D}>\kappa_{D}^{\text {crit }}$. The analysis of the Schrödinger-like equation gives the critical point $\kappa_{D}^{\text {crit }}$, which coincides with the value in the SD equation.

We note here that the size of the "repulsive centrifugal potential" becomes significant for $D \gg 4$. This is the very reason why we obtain $\kappa_{D}^{\text {crit }}$ larger than the NDA estimate.

We next comment on the case with non-running $g_{D}$ [37. In this case, the "potential" $V(r)$ is given by

$$
\begin{aligned}
V(r) & \equiv-(D-1) g_{D}^{2} \int \frac{d^{D} p_{E}}{(2 \pi)^{D}} \frac{e^{i p_{E} \cdot u}}{p_{E}^{2}} \\
& =-(D-1) \frac{\Gamma(D / 2-1)}{4 \pi^{D / 2}} \frac{g_{D}^{2}}{r^{D-2}}
\end{aligned}
$$

in the Landau gauge $(\xi=0)$. When the potential behaves as $-1 / r^{s}(0<s<2)$ for sufficiently large $r$, the spectrum contains a countably infinite number of bound states [39]. The dynamical symmetry breaking thus occurs for any value of the gauge coupling in $2<D<4$ [37]. However, it is not true in $D>4$. There is a critical point and the scaling relation is power-like in the numerical analysis for $D=5,6$ 24]. 


\section{APPENDIX C: EFFECTS OF IR CUTOFF IN THE GAP EQUATION AT THE FIXED POINT}

In this Appendix we solve the linearized equation (37) in the presence of the IR cutoff $M_{0} \sim R^{-1}$. The integral gap equation is written as the differential one

$$
\frac{d^{2} B(x)}{d x^{2}}+\frac{2 \omega+1}{x} \frac{d B(x)}{d x}+\omega^{2}\left(1+\nu^{2}\right) \frac{B(x)}{x\left(x+B_{0}^{2}\right)}=0,
$$

with two (infrared and ultraviolet) boundary conditions

$$
\begin{aligned}
& \left.x^{2 \omega+1} \frac{d}{d x} B(x)\right|_{x=M_{0}^{2}}=0, \quad(\text { IR-BC }) \\
& \left.\left(1+\frac{x}{2 \omega} \frac{d}{d x}\right) B(x)\right|_{x=\Lambda^{2}}=m_{0}, \quad(\mathrm{UV}-\mathrm{BC})
\end{aligned}
$$

(hereafter, we consider the chiral limit $m_{0}=0$ ).

The general solution of Eq.(C1) has the form

$$
B_{0}(x) / B_{0}=C_{1} u_{1}(x)+C_{2} u_{2}(x),
$$

where as for two independent solutions of the differential equation we take

$$
\begin{aligned}
& u_{1}(x) \equiv F\left(\omega(1+i \nu), \omega(1-i \nu), 1+2 \omega ;-\frac{x}{B_{0}^{2}}\right) \\
& u_{2}(x) \equiv\left(\frac{x}{B_{0}^{2}}\right)^{-\omega(1+i \nu)} F\left(\omega(1+i \nu),-\omega(1-i \nu), 1+2 i \omega \nu ;-\frac{B_{0}^{2}}{x}\right)+\text { c.c. }
\end{aligned}
$$

(we consider the case $\kappa_{D}>\kappa_{D}^{\text {crit }}$ ).

The boundary conditions (C2), C3) lead to the following equation determining the mass spectrum:

$$
\phi=A_{1} B_{2}-A_{2} B_{1}=0,
$$

where we defined the functions $A_{i}, B_{i}$ as

$$
A_{i}=\left.\left(1+\frac{1}{2 \omega} x \frac{d}{d x}\right) u_{i}(x)\right|_{x=\Lambda^{2}}, \quad B_{i}=\left.x \frac{d u_{i}(x)}{d x}\right|_{x=M_{0}^{2}} .
$$

Using the formulas for differentiating hypergeometric functions [40], the functions $A_{i}, B_{i}$ can be recasted as

$$
\begin{gathered}
A_{1}=F\left(\omega(1+i \nu), \omega(1-i \nu), 2 \omega ;-\frac{\Lambda^{2}}{B_{0}^{2}}\right), \\
A_{2}=\operatorname{Re}\left[(1-i \nu)\left(\frac{B_{0}^{2}}{\Lambda^{2}}\right)^{\omega(1+i \nu)} F\left(\omega(1+i \nu), 1-\omega(1-i \nu), 1+2 i \omega \nu ;-\frac{B_{0}^{2}}{\Lambda^{2}}\right)\right] \\
B_{1}=-\frac{\omega^{2}\left(1+\nu^{2}\right)}{1+2 \omega} \frac{M_{0}^{2}}{B_{0}^{2}} F\left(1+\omega(1+i \nu), 1+\omega(1-i \nu), 2+2 \omega ;-\frac{M_{0}^{2}}{B_{0}^{2}}\right), \\
B_{2}=-2 \omega \operatorname{Re}\left[(1+i \nu)\left(\frac{B_{0}^{2}}{M_{0}^{2}}\right)^{\omega(1+i \nu)} F\left(1+\omega(1+i \nu),-\omega(1-i \nu), 1+2 i \omega \nu ;-\frac{B_{0}^{2}}{M_{0}^{2}}\right)\right] .
\end{gathered}
$$


Since we always assume that $B_{0} / \Lambda \ll 1$ we can use for $A_{i}$ their asymptotic expressions

$$
\begin{aligned}
& A_{1} \simeq\left|c_{0}\right| \sqrt{1+\nu^{2}}\left(\frac{B_{0}^{2}}{\Lambda^{2}}\right)^{\omega} \sin \left(\omega \nu \ln \frac{\Lambda^{2}}{B_{0}^{2}}+\tan ^{-1} \nu+\theta\right), \\
& A_{2} \simeq \sqrt{1+\nu^{2}}\left(\frac{B_{0}^{2}}{\Lambda^{2}}\right)^{\omega} \cos \left(\omega \nu \ln \frac{\Lambda^{2}}{B_{0}^{2}}+\tan ^{-1} \nu\right)
\end{aligned}
$$

where $c_{0}$ and $\theta$,

$$
\theta=\arg \frac{\Gamma(1+2 i \omega \nu)}{\Gamma(\omega(1+i \nu)) \Gamma(1+\omega(1+i \nu))},
$$

are defined after Eqs. (46), (47). For the function $B_{1}$ we use the formula (2.10.2) from 40 in order to rewrite it in the form similar to $B_{2}$, thus we have

$$
\begin{aligned}
B_{1}= & 2 \omega \sqrt{1+\nu^{2}}\left|c_{0}\right|\left(\frac{B_{0}^{2}}{M_{0}^{2}}\right)^{\omega} \operatorname{Im}\left\{\left(\frac{B_{0}^{2}}{M_{0}^{2}}\right)^{i \omega \nu} e^{-i \theta+i \tan ^{-1} \nu}\right. \\
& \left.\times F\left(1+\omega(1+i \nu), \omega(-1+i \nu), 1+2 i \omega \nu ;-\frac{B_{0}^{2}}{M_{0}^{2}}\right)\right\}, \\
B_{2}= & -2 \omega \sqrt{1+\nu^{2}}\left(\frac{B_{0}^{2}}{M_{0}^{2}}\right)^{\omega} \operatorname{Re}\left\{\left(\frac{B_{0}^{2}}{M_{0}^{2}}\right)^{i \omega \nu} e^{i \tan ^{-1} \nu}\right. \\
& \left.\times F\left(1+\omega(1+i \nu), \omega(-1+i \nu), 1+2 i \omega \nu ;-\frac{B_{0}^{2}}{M_{0}^{2}}\right)\right\} .
\end{aligned}
$$

Combining Eqs. C14 $,(\mathrm{C} 14),(\mathrm{C} 16),(\mathrm{C} 17)$, the gap equation is transformed to the form

$$
\begin{aligned}
\phi \simeq & -2 \omega\left|c_{0}\right|\left(1+\nu^{2}\right) \cos \theta\left(\frac{M_{0}^{2}}{\Lambda^{2}}\right)^{\omega}\left(\frac{B_{0}^{2}}{M_{0}^{2}}\right)^{2 \omega} \\
& \times \operatorname{Im}\left[\left(\frac{\Lambda^{2}}{M_{0}^{2}}\right)^{i \omega \nu} e^{2 i \tan ^{-1} \nu} F\left(1+\omega(1+i \nu), \omega(-1+i \nu), 1+2 i \omega \nu ;-\frac{B_{0}^{2}}{M_{0}^{2}}\right)\right]=0 .
\end{aligned}
$$

One can convince oneself that for $M_{0} \ll B_{0}$ the last equation is equivalent to

$$
\sin \left(\omega \nu \ln \frac{\Lambda^{2}}{B_{0}^{2}}+\theta+\tan ^{-1} \nu\right)=0
$$

which gives solutions (49). On the other hand, for $B_{0} \ll M_{0}$ we can use a power expansion of the hypergeometric function to get the equation for the dynamical mass near the phase transition point:

$$
\begin{aligned}
& \sin \left(\omega \nu \ln \frac{\Lambda^{2}}{M_{0}^{2}}+2 \tan ^{-1} \nu\right)+\omega\left[\frac{\left(1+\nu^{2}\right)\left((1+\omega)^{2}+\omega^{2} \nu^{2}\right)}{1+4 \omega^{2} \nu^{2}}\right]^{1 / 2} \frac{B_{0}^{2}}{M_{0}^{2}} \\
& \times \sin \left(\omega \nu \ln \frac{\Lambda^{2}}{M_{0}^{2}}-\tan ^{-1} 2 \omega \nu+\tan ^{-1} \frac{\omega \nu}{1+\omega}+\tan ^{-1} \nu\right)=0 .
\end{aligned}
$$

The nontrivial solution for the dynamical mass arises when $\nu \equiv \sqrt{\kappa_{D} / \kappa_{D}^{\text {crit }}-1}$ exceeds the critical value determined by the equation

$$
\omega \nu_{c} \ln \frac{\Lambda^{2}}{M_{0}^{2}}+2 \tan ^{-1} \nu_{c}=\pi
$$


It should be noted that $\nu_{c}$ is a small number

$$
\nu_{c} \simeq \frac{\pi}{2+\omega \ln \frac{\Lambda^{2}}{M_{0}^{2}}} \ll 1,
$$

for $\Lambda \gg M_{0}$. Note also that the form of the gap equation ( $\left.\overline{\mathrm{C} 18}\right)$ is different in two regions $M_{0} \ll B_{0}$ and $M_{0} \gg B_{0}$ : while in the first one $\left(M_{0} \ll B_{0}\right)$ we observe oscillations in the mass variable, in the second one $\left(M_{0} \gg B_{0}\right)$ such oscillations disappear. This is reflected in the character of the mass dependence on the coupling constant (compare Eqs. (54) and (C24) below). In general, it can be shown that Eq. (C18) has $n$ nontrivial solutions where the number $n$ is given by

$$
n=\left[\pi^{-1} \omega \nu \ln \frac{\Lambda^{2}}{M_{0}^{2}}+2 \tan ^{-1} \nu\right]
$$

and the symbol $[C]$ means the integer part of the number $C$.

Near the critical point we thus obtain the mean-field scaling relation for the dynamical mass

$$
\frac{B_{0}^{2}}{M_{0}^{2}}=\frac{\pi}{\omega\left(2 \omega^{2}+2 \omega+1\right)} \frac{\nu-\nu_{c}}{\nu_{c}^{2}}, \quad \nu \gtrsim \nu_{c}, \quad \nu_{c} \ll 1,
$$

which is cited in Eq. (60).

\section{APPENDIX D: FORMULAS OF THE EFFECTIVE GAUGE COUPLING STRENGTH}

The relation between effective and $\overline{\mathrm{MS}}$ couplings $(D=4+2)$ is given by Eq.(72) where the terms $K_{g}$, $K_{b}, K_{f}$ denote one-loop contributions from gauge bosons, gauge scalars, and fermions, respectively. The formulas of $K_{g}, K_{b}$, and $K_{f}$ are given by

$$
\begin{aligned}
& K_{g}\left(q^{2}, \Lambda_{g}^{2}\right) \equiv 4 C_{G}\left(\frac{5}{18}+\frac{1}{6} \ln \frac{\Lambda_{g}^{2}}{\left(-q^{2}\right)}+\frac{\Lambda_{g}^{2}}{\left(-q^{2}\right)} \tilde{K}_{g}\left(q^{2}, \Lambda_{g}^{2}\right)\right), \\
& K_{b}\left(q^{2}, \Lambda_{g}^{2}\right) \equiv-2 C_{G}\left(\frac{31}{450}+\frac{1}{30} \ln \frac{\Lambda_{g}^{2}}{\left(-q^{2}\right)}+\frac{\Lambda_{g}^{2}}{\left(-q^{2}\right)} \tilde{K}_{b}\left(q^{2}, \Lambda_{g}^{2}\right)\right), \\
& K_{f}\left(q^{2}, \Lambda_{g}^{2}\right) \equiv-2 \eta T_{R} N_{f}\left(\frac{47}{900}+\frac{1}{30} \ln \frac{\Lambda_{g}^{2}}{\left(-q^{2}\right)}+\frac{\Lambda_{g}^{2}}{\left(-q^{2}\right)} \tilde{K}_{f}\left(q^{2}, \Lambda_{g}^{2}\right)\right),
\end{aligned}
$$




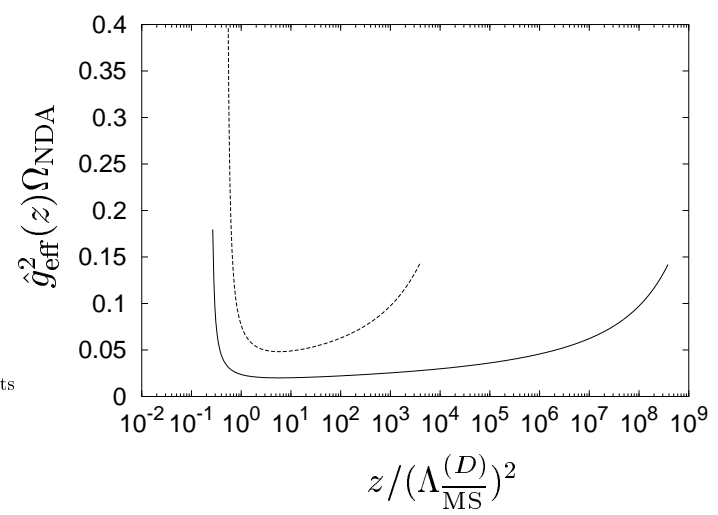

FIG. 18: The effective coupling in the strongly interacting phase $\left(\left(\Lambda_{\overline{\mathrm{MS}}}^{(D)}\right)^{2}>0\right)$. The solid and dashed lines represent the graphs for $\left(\Lambda_{g} R\right)^{2}=4 \times 10^{10}, 4 \times 10^{5}$ with $N_{f}=2, N=3,\left(\Lambda_{\overline{\mathrm{MS}}}\right)^{2}=10^{2}$, respectively.

where the sum of the KK-modes is approximated by replacing it to an corresponding integral. The functions $\tilde{K}_{i}(i=g, b, f)$ are defined by

$$
\begin{aligned}
\tilde{K}_{g}\left(q^{2}, \Lambda_{g}^{2}\right) \equiv & \int_{0}^{1} d x f\left(q^{2}, \Lambda_{g}^{2}, x\right), \\
= & -\frac{4}{3}+\frac{5}{18} \frac{q^{2}}{\Lambda_{g}^{2}}+\frac{1}{3}\left(4-\frac{q^{2}}{\Lambda_{g}^{2}}\right)^{3 / 2}\left(\frac{\Lambda_{g}^{2}}{-q^{2}}\right)^{1 / 2} \operatorname{arctanh} \sqrt{\frac{-q^{2}}{4 \Lambda_{g}^{2}-q^{2}}}, \\
\tilde{K}_{b}\left(q^{2}, \Lambda_{g}^{2}\right) \equiv & \int_{0}^{1} d x(2 x-1)^{2} f\left(q^{2}, \Lambda_{g}^{2}, x\right), \\
= & \frac{16}{15} \frac{\Lambda_{g}^{2}}{q^{2}}-\frac{28}{45}+\frac{31}{450} \frac{q^{2}}{\Lambda_{g}^{2}}+\frac{1}{15}\left(4-\frac{q^{2}}{\Lambda_{g}^{2}}\right)^{5 / 2}\left(\frac{\Lambda_{g}^{2}}{-q^{2}}\right)^{3 / 2} \operatorname{arctanh} \sqrt{\frac{-q^{2}}{4 \Lambda_{g}^{2}-q^{2}}}, \\
\tilde{K}_{f}\left(q^{2}, \Lambda_{g}^{2}\right) \equiv & \int_{0}^{1} d x x(1-x) f\left(q^{2}, \Lambda_{g}^{2}, x\right), \\
= & -\frac{4}{15} \frac{\Lambda_{g}^{2}}{q^{2}}-\frac{8}{45}+\frac{47}{900} \frac{q^{2}}{\Lambda_{g}^{2}} \\
& -\frac{1}{15}\left(4-\frac{q^{2}}{\Lambda_{g}^{2}}\right)^{3 / 2}\left(1+\frac{q^{2}}{\Lambda_{g}^{2}}\right)\left(\frac{\Lambda_{g}^{2}}{-q^{2}}\right)^{3 / 2} \operatorname{arctanh} \sqrt{\frac{-q^{2}}{4 \Lambda_{g}^{2}-q^{2}}},
\end{aligned}
$$

with $f$ being

$$
f\left(q^{2}, \Lambda_{g}^{2}, x\right) \equiv\left(1-x(1-x) \frac{q^{2}}{\Lambda_{g}^{2}}\right) \ln \left(1-\frac{q^{2}}{\Lambda_{g}^{2}} x(1-x)\right)
$$

We next discuss behavior of the effective coupling $\hat{g}_{\text {eff }}^{2}(z)$ in the energy region $z \ll \Lambda^{2}$. Expanding the function $f$ around $q^{2}=0$

$$
f\left(q^{2}, \Lambda_{g}^{2}, x\right)=-x(1-x) \frac{q^{2}}{\Lambda_{g}^{2}}+\frac{1}{2} x^{2}(1-x)^{2} \frac{q^{4}}{\Lambda_{g}^{4}}+\mathcal{O}\left(\left(\frac{q^{2}}{\Lambda_{g}^{2}}\right)^{3}\right),
$$


we find

$$
\begin{aligned}
& \tilde{K}_{g}\left(q^{2}, \Lambda_{g}^{2}\right)=-\frac{1}{6} \frac{q^{2}}{\Lambda_{g}^{2}}+\frac{1}{60}\left(\frac{q^{2}}{\Lambda_{g}^{2}}\right)^{2}+\mathcal{O}\left(\left(\frac{q^{2}}{\Lambda_{g}^{2}}\right)^{3}\right), \\
& \tilde{K}_{b}\left(q^{2}, \Lambda_{g}^{2}\right)=-\frac{1}{30} \frac{q^{2}}{\Lambda_{g}^{2}}+\frac{1}{420}\left(\frac{q^{2}}{\Lambda_{g}^{2}}\right)^{2}+\mathcal{O}\left(\left(\frac{q^{2}}{\Lambda_{g}^{2}}\right)^{3}\right), \\
& \tilde{K}_{f}\left(q^{2}, \Lambda_{g}^{2}\right)=-\frac{1}{30} \frac{q^{2}}{\Lambda_{g}^{2}}+\frac{1}{280}\left(\frac{q^{2}}{\Lambda_{g}^{2}}\right)^{2}+\mathcal{O}\left(\left(\frac{q^{2}}{\Lambda_{g}^{2}}\right)^{3}\right) .
\end{aligned}
$$

It is then easy to obtain

$$
\sum_{i=g, b, f} K_{i}\left(-z, \Lambda_{g}^{2}\right)=\left(\frac{3}{5} C_{G}-\frac{\eta}{15} T_{R} N_{f}\right) \ln \frac{\Lambda_{g}^{2}}{z}+\frac{1}{75}\left(118 C_{G}-\frac{77}{6} \eta T_{R} N_{f}\right)+\mathcal{O}\left(\frac{z}{\Lambda_{g}^{2}}\right) .
$$

Plugging Eq.(D7) into Eq.(74), we find

$$
\frac{1}{\hat{g}_{\mathrm{eff}}^{2}(z)}=-\frac{1}{g_{*}^{2}} \frac{\left(\Lambda_{\mathrm{MS}}^{(D)}\right)^{2}}{z}+\frac{1}{(4 \pi)^{3}}\left(\frac{3}{5} C_{G}-\frac{\eta}{15} T_{R} N_{f}\right) \ln \frac{\Lambda_{g}^{2}}{z}+\cdots .
$$

Note here that the effective coupling $\hat{g}_{\text {eff }}^{2}$ depends on the ultraviolet cutoff $\Lambda_{g}$, indicating the violation of the decoupling theorem.

It is now easy to see that the effective coupling $\hat{g}_{\text {eff }}^{2}$ remains finite in the infrared region $0 \leq z \ll \Lambda_{g}^{2}$ for $\left(\Lambda_{\mathrm{MS}}^{(D)}\right)^{2}<0$ for $C_{G}>\eta T_{R} N_{f} / 9$, which is automatically satisfied when $-b^{\prime}=\left(10 C_{G}-\eta T_{R} N_{f}\right) / 3>$ 0 . On the other hand, Fig. 18 shows typical behaviors of the effective coupling with $\left(\Lambda \frac{(D)}{\mathrm{MS}}\right)^{2}>0$. We find that the effective coupling diverges at $z \sim\left(\Lambda \frac{(D)}{\mathrm{MS}}\right)^{2}>0$ in this case.

[1] N. Arkani-Hamed, S. Dimopoulos, and G. Dvali, Phys. Lett. B 429, 263 (1998); Phys. Rev. D 59, 086004 (1999); I. Antoniadis, N. Arkani-Hamed, S. Dimopoulos, and G. Dvali, Phys. Lett. B 436, 257 (1998).

[2] I. Antoniadis, Phys. Lett. B 246, 377 (1990).

[3] N. Arkani-Hamed, A. G. Cohen, and H. Georgi, Phys. Rev. Lett. 86, 4757 (2001).

[4] C. T. Hill, S. Pokorski, and J. Wang, Phys. Rev. D64, 105005 (2001).

[5] M. Hashimoto, M. Tanabashi and K. Yamawaki, Phys. Rev. D64, 056003 (2001).

[6] V. A. Miransky, M. Tanabashi, and K. Yamawaki, Phys. Lett. B 221, 177 (1989); Mod. Phys. Lett. A 4, 1043 (1989).

[7] Y. Nambu, Enrico Fermi Institute Report No. 89-08, 1989; in Proceedings of the 1989 Workshop on Dynamical Symmetry Breaking, edited by T. Muta and K. Yamawaki (Nagoya University, Nagoya, Japan, 1990).

[8] W. A. Bardeen, C. T. Hill, and M. Lindner, Phys. Rev. D 41, 1647 (1990).

[9] N. Arkani-Hamed, H.-C. Cheng, B. A. Dobrescu and L. J. Hall, Phys. Rev. D62, 096006 (2000).

[10] B. A. Dobrescu, Phys. Lett. B 461, 99 (1999); hep-ph/9903407; H. Cheng, B. A. Dobrescu, and C. T. Hill, Nucl. Phys. B 589, 249 (2000).

[11] C.T. Hill, Phys. Lett. B 266, 419 (1991); 345, 483 (1995); K.Lane, and E.Eichten, Phys. Lett. B352, 382 (1995); B. A. Dobrescu and C. T. Hill, Phys. Rev. Lett. 81, 2634 (1998).

[12] A.B. Kobakhidze, Phys. Atom. Nucl. 64, 941 (2001); Yad. Fiz. 64, 1010 (2001).

[13] V. A. Miransky, Sov. J. Nucl. Phys. 38, 280 (1983); K. Higashijima, Phys. Rev. D29, 1228 (1984).

[14] K. R. Dienes, E. Dudas and T. Gherghetta, Phys. Lett. B436, 55 (1998); Nucl. Phys. B537, 47 (1999); I. Antoniadis, S. Dimopoulos, A. Pomarol and M. Quiros, Nucl. Phys. B544, 503 (1999); Z. Kakushadze, Nucl. Phys. B548, 205 (1999).

[15] H. Abe, H. Miguchi and T. Muta, Mod. Phys. Lett. A 15, 445 (2000); H. Abe, K. Fukazawa and T. Inagaki, hep-ph/0107125. 
[16] V.A. Miransky and K. Yamawaki, Phys. Rev. D 55 (1997) 5051; ibid. D 56, 3768 (1997).

[17] B. Holdom, Phys. Lett. B 150, 301 (1985); K. Yamawaki, M. Bando, and K. Matumoto, Phys. Rev. Lett. 56, 1335 (1986); T. Akiba and T. Yanagida, Phys. Lett. B 169, 432 (1986); T. Appelquist, D. Karabali, and L. C. R. Wijewardhana, Phys. Rev. Lett. 57, 957 (1986).

[18] T. Kugo and M. G. Mitchard, Phys. Lett. B282, 162 (1992).

[19] H. Georgi, E. H. Simmons, and A. G. Cohen, Phys. Lett. B236, 183 (1990).

[20] E. H. Simmons, Phys. Rev. D42, 2933 (1990).

[21] K.-I. Kondo, T. Ebihara, T. Iizuka, and E. Tanaka, Nucl. Phys. B434, 85 (1995).

[22] V. A. Miransky, Nuovo Cimento, 90 A, 149 (1985).

[23] J. M. Cornwall, R. Jackiw, and E. Tomboulis, Phys. Rev. D10, 2428 (1974); For a review, see, R. Haymaker, Riv. Nuovo Cim. 14, No. 8 , 1 (1991).

[24] K.-I. Kondo and H. Nakatani, Mod. Phys. Lett. A4, 2155 (1989).

[25] A. Manohar and H. Georgi, Nucl. Phys. B234, 189 (1984); Z. Chacko, M.A. Luty, and E. Pontón, J. High Energy Phys. 07, 036 (2000).

[26] S. Ejiri, J. Kubo and M. Murata, Phys. Rev. D62, 105025 (2000).

[27] D. Atkinson, J. Math. Phys. 28, 2494 (1987).

[28] See, for example, V. A. Miransky, Dynamical Symmetry Breaking in Quantum Field Theories (World Scientific Pub. Co., Singapore 1993).

[29] V. P. Gusynin and V. A. Miransky, Phys. Lett. B 198, 79 (1987).

[30] T. Nonoyama and M. Tanabashi, Prog. Theor. Phys. 81, 209 (1989).

[31] H. Pagels and S. Stokar, Phys. Rev. D 20, 2947 (1979).

[32] K-I. Kondo, in New Trends to Strong Coupling Gauge Theories, Nagoya, August 24-27, ed. by M. Bando, T. Muta and K. Yamawaki (Nagoya University, Nagoya, Japan 1988); K-I. Kondo, Y. Kikukawa, and H. Mino, Phys. Lett. B220, 270 (1989); V. P. Gusynin, Mod. Phys. Lett. A5, 133 (1990).

[33] V. A. Miransky, Int. J. Mod. Phys. A 8, 135 (1993).

[34] V. A. Miransky, I. A. Shovkovy and L. C. Wijewardhana, Phys. Lett. B468, 270 (1999).

[35] P. I. Fomin, V. P. Gusynin, V. A. Miransky, and Yu. A. Sitenko, Riv. Nuovo Cim. 6, No. 5 , 1 (1983).

[36] V. A. Miransky, V. P. Gusynin, and Yu. A. Sitenko, Phys. Lett. 100B, 157 (1981).

[37] V. P. Gusynin, A. W. Schreiber, T. Sizer and A. G. Williams, Phys.Rev.D60, 065007 (1999).

[38] See, for example, P. M. Morse and H. Feshbach, Methods of Theoretical Physics (McGraw-Hill, New York, 1953), Ch.12 $§ 12.3$ pp.1665-1667.

[39] R. Courant and D. Hilbert, Methods of Mathematical Physics (Wiley-Interscience Pub., New York, 1953) Ch.VI. $\S 5$.

[40] H. Bateman and A. Erdelyi, Higher Transcendental Functions, vol. 1, McGraw-Hill, New York (1953). 